\title{
Depletion of the Third Complement Component Ameliorates Age- Dependent Oxidative Stress and Positively Modulates Autophagic Activity in Aged Retinas in a Mouse Model
}

\author{
Dorota Rogińska, ${ }^{1}$ Miłosz P. Kawa, ${ }^{1}$ Ewa Pius-Sadowska, ${ }^{1}$ Renata Lejkowska, ${ }^{1}$ \\ Karolina Luczkowska, ${ }_{1}$ Barbara Wiszniewska, ${ }^{2}$ Kai Kaarniranta, ${ }^{3,4}$ Jussi J. Paterno, ${ }^{3,4}$ \\ Christian A. Schmidt, ${ }^{5}$ Bogusław Machaliński, ${ }^{1}$ and Anna Machalińska ${ }^{2,6}$ \\ ${ }^{1}$ Department of General Pathology, Pomeranian Medical University, Al. Powstancow Wlkp. 72, 70-111 Szczecin, Poland \\ ${ }^{2}$ Department of Histology and Embryology, Pomeranian Medical University, Al. Powstancow Wlkp. 72, 70-111 Szczecin, Poland \\ ${ }^{3}$ Department of Ophthalmology, Institute of Clinical Medicine, University of Eastern Finland, 70211 Kuopio, Finland \\ ${ }^{4}$ Department of Ophthalmology, Kuopio University Hospital, 70211 Kuopio, Finland \\ ${ }^{5}$ Clinic for Internal Medicine C, University of Greifswald, 17475 Greifswald, Germany \\ ${ }^{6}$ Department of Ophthalmology, Pomeranian Medical University, Al. Powstancow Wlkp. 72, 70-111 Szczecin, Poland
}

Correspondence should be addressed to Anna Machalińska; annam@pum.edu.pl

Received 25 April 2017; Revised 28 June 2017; Accepted 9 July 2017; Published 8 August 2017

Academic Editor: Kota V. Ramana

Copyright (C) 2017 Dorota Rogińska et al. This is an open access article distributed under the Creative Commons Attribution License, which permits unrestricted use, distribution, and reproduction in any medium, provided the original work is properly cited.

\begin{abstract}
The aim of the study was to investigate the influence of complement component C3 global depletion on the biological structure and function of the aged retina. In vivo morphology (OCT), electrophysiological function (ERG), and the expression of selected oxidative stress-, apoptosis-, and autophagy-related proteins were assessed in retinas of 12-month-old C3-deficient and WT mice. Moreover, global gene expression in retinas was analyzed by RNA arrays. We found that the absence of active C3 was associated with (1) alleviation of the age-dependent decrease in retinal thickness and gradual deterioration of retinal bioelectrical function, (2) significantly higher levels of antioxidant enzymes (catalase and glutathione reductase) and the antiapoptotic survivin and Mcl-1/Bak dimer, (3) lower expression of the cellular oxidative stress marker-4HNE-and decreased activity of proapoptotic caspase-3, (4) ameliorated retinal autophagic activity with localization of ubiquitinated protein conjugates commonly along the retinal pigment epithelium (RPE) layer, and (5) significantly increased expression of several gene sets associated with maintenance of the physiological functions of the neural retina. Our findings shed light on mechanisms of age-related retinal alterations by identifying C3 as a potential therapeutic target for retinal aging.
\end{abstract}

\section{Introduction}

Age-related deterioration of the retina seriously constrains the vision quality of a growing number of elderly people worldwide. Recently, the dysregulation of inflammatory and immune pathways has become increasingly accepted as key fundamental mechanisms of age-related retinal alterations [1].
It is widely accepted that age-related retinal cell injury caused by accumulative oxidative stress (OS) represents an initial determinant for various age-related retinal malfunctions. Lipofuscin, the aging-associated pigmented material, which accumulates in the cells of the retinal pigment epithelium (RPE) with age together with the accumulation of basal laminar deposits, and drusen in Bruch's membrane have been proposed to be mainly responsible for the increase in 
local oxidative stress in the retina [2]. Oxidative stress-related cellular products develop as a consequence of autophagy dysregulation in RPE cells. A large amount of evidence indicates that autophagy declines with age and that this progressive reduction may have a causative role in the development of age-related retinal alterations [3]. Impaired autophagy in the RPE leads to cell transcytosis and exocytosis and early signs of retinal degeneration [4]. Abnormal autophagic and ubiquitin-proteasome pathway (UPP) cleansing has been documented in aged RPE cells and in retinas from AMD patients [3-5].

Insufficient degradation of toxic proteins and oxidative lipids triggers an immune response in the affected tissues. In the elderly, the combination of increased production of reactive oxygen species (ROS) and decreased antioxidant functions, accompanied by an upregulation of several inflammatory genes, such as those encoding interleukin- (IL-) $1 \beta$, IL-6, IL-8, and tumor necrosis factor (TNF), leads to a bidirectional attack, both from the altered redox status and from the dysregulated immune responses [6]. Compelling evidence suggests that the complement system (CS) plays a critical role in regulating inflammatory and immunological processes in aged retinas. In humans, it was found that C3 gene expression is upregulated with aging [7]. Likewise, aged CD59-deficient animals exhibit increased expression of several activators of the alternative CS pathway (C3, CFB, and CFD), particularly in the RPE-choroid layer [8]. Thus, dysregulation of the complement system has been implicated in the pathogenesis of age-related retinal disorders, for example, AMD. Consequently, chronic and repetitive nonlethal RPE injuries, together with an oxidative environment and autophagy dysregulation, appear to be important factors for the development of age-related retinal changes.

Although there is a significant body of research on the mechanisms of retinal aging, the complex pathways between oxidative stress, initial cellular damage, locally triggered inflammation, autophagy, and UPP conditions are not yet apparent. Therefore, the aims of this study were twofold: (1) to investigate the effect of $\mathrm{C} 3$ depletion on oxidative metabolism, apoptosis, and autophagic and proteasomal cleansing in aged retinal tissue in vivo and (2) to investigate the effect of $\mathrm{C} 3$ deficiency on global gene expression in aging retinal tissue.

\section{Materials and Methods}

2.1. Animals. Pathogen-free 12-week-old mature C3deficient (C3-/-) female mice (strain: B6;129S4-C3tm1Crr/ J; Jackson Laboratory, Bar Harbor, ME, USA) and agematched WT C57BL/6 mice (Polish Academy of Sciences, Wroclaw, Poland) were purchased and maintained under standard conditions with a 12-hour day (160-lux)-night cycle and with ad libitum feeding. These C3-/- mice produce no detectable C3 protein and no complement activity. Wildtype age- and gender-matched C57BL/6 littermates were used as the C3-positive controls. Each mouse strain was divided into two groups, and one group was maintained to an older age of 12 months. These animals were then used in the indicated experiments. All animal procedures were performed according to the regulations in the ARVO Statement for the Use of Animals in Ophthalmic and Vision Research and were approved by the local ethics committee. We followed the methods of Machalińska et al. [9].

2.2. Electroretinography. Scotopic and photopic ERGs were recorded from 3- and 12-month-old C3-deficient and WT mice ( $n=10$ mice, for each group of animals). Following overnight dark adaptation, the mice were anesthetized by an intraperitoneal injection of ketamine $(40 \mathrm{mg} / \mathrm{kg})$ and xylazine $(4 \mathrm{mg} / \mathrm{kg})$ (both from Biowet, Puławy, Poland). Then, the cornea was anesthetized (Alcaine; Alcon, Fort Worth, TX, USA), and the pupils were dilated with $1 \%$ atropine (Polfa Warszawa, Poland). Retinal responses were recorded with gold ring contact electrodes (LKC Technologies, Gaithersburg, MD, USA). Needle electrodes were placed under the scalp between the eyes and in the tail as the reference and ground leads, respectively. ERGs were differentially amplified (0.05-1500 Hz), averaged, and stored using an LKC UTAS BigShot system. ERGs were recorded in response to strobe flash stimuli presented in the LKC Ganzfeld bowl using a protocol similar to that used for human testing. To assess rod photoreceptor function, a strobe white-flash stimulus was presented to the darkadapted dilated eye with a low flash intensity $(24 \mathrm{~dB}$ attenuation), and 8 responses recorded at intervals of $8 \mathrm{~s}$ were computer-averaged. Mixed rod and cone responses were obtained by stimulation with white flashes of maximum intensity equal to approximately $1.6 \mathrm{~cd}^{*} \mathrm{~s} / \mathrm{m}^{2}$ (standard flash, SF; $0 \mathrm{~dB}$ attenuation). The retinal responses were measured twice with a $28 \mathrm{~s}$ interstimulus interval and averaged. To evaluate the function of the cone photoreceptors, the animals were light-adapted for $10 \mathrm{~min}$ under a white background $\left(32 \mathrm{~cd} / \mathrm{m}^{2}\right)$. Next, a strobe white-flash stimulus was presented to the dilated eye in the Ganzfeld bowl using the maximum flash intensity ( $0 \mathrm{~dB}$ attenuation), and responses to 8 flashes with an interstimulus interval of $1 \mathrm{~s}$ were recorded and averaged. The amplitude of the b-wave was measured from the a-wave trough to the peak of the b-wave or, if an a-wave was not present, from the prestimulus baseline to the peak of the b-wave. To demonstrate changes in the retinal bioelectrical response over time, the data for each individual 1-year-old mouse were calculated as a percentage value in reference to the b-wave amplitude measurements obtained from 3-month-old mice (which was considered 100\%).

2.3. SD-OCT Imaging. Spectral domain optical coherence tomography was performed in 12-week-old and 12-monthold C3-deficient and WT mice ( $n=10$ mice, for both groups of animals). For the imaging procedures, the mice were anesthetized by intraperitoneal injection of ketamine $(40 \mathrm{mg} / \mathrm{kg})$ and xylazine $(4 \mathrm{mg} / \mathrm{kg})$. The pupils were dilated with $1 \%$ atropine. Artificial tears were used throughout the procedure to maintain corneal clarity. Images were obtained using the Envisu R2200-HR SD-OCT device (Bioptigen Inc., Durham, NC, USA) with the reference arm placed at approximately $1197 \mathrm{~mm}$. SD-OCT images of a specific region of each eye were obtained using the optic disc as a landmark. 
Rectangular scans $(1.5 \mathrm{~mm} \times 1.5 \mathrm{~mm}, 1280$ A-scans/Bscan $\times 150$ B-scans) were obtained first while centering on the optic nerve and then with the nerve displaced either temporally/nasally or superiorly/inferiorly. For quantitative analysis, the distance between the first high reflective layer (i.e., the vitreoretinal interface) and the retinal pigment epithelium was measured using InVivoVue software. If the optic nerve was placed temporally/nasally, three B-scans (at the level of the nerve, $450 \mu \mathrm{m}$ up and down from the nerve) were added with one measurement performed $750 \mu \mathrm{m}$ away from the optic disc, on each side. When the optic nerve was placed superiorly/inferiorly, one B-scan was placed $750 \mu \mathrm{m}$ away from the optic disc with 3 measurements (at the level of the nerve, $450 \mu \mathrm{m}$ to the left and right of the nerve) for both positions. The mean value of all measurements was considered the total retinal thickness. To demonstrate changes in the total thickness of the retina over time, the data are presented as a percentage value calculated for each individual 1-yearold mouse in reference to measurements obtained from 3-month-old mice (which was considered 100\%).

2.4. Tissue Collection. At the end of the experiment, 12month-old C3-deficient and WT mice were sacrificed by cervical dislocation under anesthesia. The eyes were enucleated and divided for different assessments. For histological and immunofluorescence analyses, the tissue was fixed in Davidson's solution, embedded in paraffin, and cut into $5 \mu \mathrm{m}$ thick sections ( $n=3$ eyes in each group of animals). For Affymetrix Gene Chip Microarray, Western blot, and Luminex analyses, total RNA and protein were isolated using the PARIS Kit (Thermo Fisher Scientific, Waltham, MA, USA) following the manufacturer's instructions ( $n=14$ eyes in each group of animals).

2.5. Histological and Immunofluorescence Analyses. For cross sections, the tissue was routinely stained with hematoxylin and eosin (Sigma-Aldrich, Saint Louis, MO, USA). For immunofluorescence analysis, sections were deparaffinized in xylene $(2 \times 15 \mathrm{~min})$ followed by hydration in solutions with decreasing ethanol concentrations $(100,95,80,75$, and $50 \%)$. In the first stage of ubiquitin detection, slides were quenched with $0.1 \mathrm{M}$ glycine in TBS for 10 minutes. After 30 minutes of blocking in IHC blocking solution (Bethyl Laboratories Inc., Montgomery, TX, USA), the sections were incubated with the primary antibody, rabbit anti-ubiquitin $(1: 200)$ (Dako Corporation, Carpinteria, CA, USA, cat. number $\mathrm{Z} 0458$ ), at $4^{\circ} \mathrm{C}$ overnight. On the next day, the secondary antibody, goat anti-rabbit-Alexa Fluor-488 (1:500), was applied followed by DAPI (dilution $1: 10000$ ) for nuclear staining. For the negative control sections, incubation with the primary antibody was omitted and the sections were stained only with the secondary antibody and DAPI solution. For C3 staining, Tyramide Signal Amplification (TSA) (Thermo Fisher Scientific, Waltham, MA, USA, cat. number T20925) protocol was applied, according to the manufacturer's instructions. Briefly, after 45 minutes of quenching with $3 \%$ $\mathrm{H}_{2} \mathrm{O}_{2}$ in PBS and 30 minutes of blocking in 1\% BSA, the sections were incubated with the primary antibody, rabbit anti-C3 (1:100) (Bioss Antibodies Inc., Woburn, MA, USA, cat. number bs-2934R), at $4^{\circ} \mathrm{C}$ overnight. On the next day, the secondary antibody, goat anti-rabbit-HRP (1:100), was applied. Fluorescence detection and signal amplification were achieved by staining the sections with Tyramide-Alexa Fluor 594 in amplification buffer $/ 0.0015 \% \mathrm{H}_{2} \mathrm{O}_{2}$ for 6 minutes, at room temperature. DAPI was used for counterstain (1:10000). The sections from 12-month-old C3-deficient animals served as a staining control. After Mowiol mounting (Calbiochem, San Diego, CA, USA), all sections were subjected to fluorescence microscopy analysis (Carl Zeiss MicroImaging, Oberkochen, Germany).

2.6. Western Blot and Densitometry Analyses. Equal amounts of proteins ( $20 \mu \mathrm{g} /$ well) were loaded and separated by $4-20 \%$ sodium dodecyl sulfate polyacrylamide gel electrophoresis (SDS-PAGE, mini-PROTEAN II electrophoresis system, Bio-Rad, Hercules, CA, USA) and then transferred to a $0.2 \mu \mathrm{m}$ polyvinylidene fluoride (PVDF) membrane (BioRad, Hercules, CA, USA). After blocking with 3\% BSA in TBST, the membrane was probed with a primary antibody as follows: rabbit anti-catalase (1:750) (Abcam, Cambridge, UK, cat. number ab16731), rabbit anti-glutathione reductase (GSR) (1:1000) (Abcam, cat number ab16801), mouse anti-4HNE (1:1000) (Abcam, cat. number ab48506), rabbit anti-LC3B (1:375) (Abcam, cat. number ab48394), mouse anti-p62 (1:500) (Abcam, cat number ab56416), rabbit anti-ubiquitin $(1: 1000)$ (Dako Corporation, Carpinteria, CA, USA, cat. number Z0458), or mouse anti- $\beta$ actin $(1: 1200)$ (Santa Cruz Biotechnology, Dallas, TE, USA, cat. number sc-47778), and incubated overnight at $4^{\circ} \mathrm{C}$. Immunoreactive bands were detected using a horseradish peroxidase-conjugated goat anti-rabbit and goat anti-mouse secondary antibody. Chemiluminescence detection was performed using the ECL Select Detection Kit (GE Healthcare, formerly Amersham Life Sciences, Little Chalfont, UK), and the bands were subsequently visualized using a UVP camera (GelDoc-It Imaging System; Bio-Rad, Hercules, CA, USA). Equal loading in the lanes was evaluated by stripping the blots for $2 \mathrm{~h}$ at $37^{\circ} \mathrm{C}$ and then overnight at room temperature (IgG Elution Buffer; Thermo Fisher Scientific, Waltham, MA, USA). Reprobing was then performed in an analogous manner with a mouse anti- $\beta$-actin as described above. The Band Analysis tool available in the ImageLab software version 4.1 was used to determine the background-subtracted density of the bands. The relative protein expression levels of $4 \mathrm{HNE}$ (4-hydroksy-2,3-nonenal), catalase, GSR, p62, and ubiquitin were quantified in comparison to those of $\beta$-actin. The LC3B relative protein expression level was calculated by estimation of the density of two bands, LC3B-I and LC3B-II, and presented as the LC3B-II/LC3B-I ratio.

2.7. Luminex Assay. Survivin, Mcl-1/Bak dimer, and active caspase-3 concentrations were quantified in C3-deficient and WT mouse retina homogenates by multiplex fluorescent bead-based immunoassays (Luminex Corporation, Austin, TX, USA) using commercial Bio-Plex Pro $^{\text {TM }}$ RBM Apoptosis Multiplex Assays, Panel 3 Analytes (Bio-Rad, Hercules, CA, USA). After adding blocking buffer to all wells of the plate, $30 \mu \mathrm{L}$ of each standard control and sample was added to 
the plate together with the multiplex antibody capture bead solution, and the plate was incubated with agitation for $1 \mathrm{hr}$ at room temperature. Subsequently, each well was washed 3 times with $100 \mu \mathrm{L}$ assay buffer using a hand-held magnet. Next, $40 \mu \mathrm{L}$ of detection antibody cocktail was pipetted into each well, and the plate was sealed and incubated at room temperature for $1 \mathrm{hr}$ on a shaker. After this step, $20 \mu \mathrm{L}$ streptavidin-phycoerythrin mixture was added to the plate which was incubated with agitation for 30 minutes in the dark. Finally, after washing, the microspheres in each well were resuspended in $100 \mu \mathrm{L}$ assay buffer and shaken at room temperature for $30 \mathrm{sec}$. The plate was then read and analyzed on the Luminex analyzer, and the analyte concentrations were determined from five different standard curves showing the MFI (median fluorescence intensity) versus protein concentration. To standardize the final concentration values, the obtained data were normalized to the total protein concentration.

2.8. Affymetrix GeneChip Microarray and Data Analysis. Total RNA isolated from four retinas obtained from 12month-old C3-deficient and WT mice were pooled to generate two samples for the subsequent experimental procedures. A sense-strand cDNA generated from the total RNA using an Ambion WT Expression Kit (Thermo Fisher Scientific, Waltham, MA, USA) was subjected to fragmentation and labeling using the GeneChip WT Terminal Labeling Kit (Affymetrix, Santa Clara, CA) and then hybridized onto an Affymetrix Mouse Gene 2.1 ST Array Strip. Hybridization and subsequent fluidics and scanning steps were performed with an Affymetrix GeneAtlas ${ }^{\mathrm{TM}}$ System. The preliminary analysis of the scanned chips was performed using Affymetrix GeneAtlas Operating Software. The quality of the gene expression data was checked according to the quality control criteria provided by the software. The obtained CEL files were imported into the downstream data analysis software. All analyses were performed using BioConductor software based on the statistical R programming language. For background correction, normalization, and summation of raw data, the Robust Multiarray Averaging (RMA) algorithm implemented in the "affy" package of BioConductor was applied. Biological annotation was obtained from the BioConductor "oligo" package in which the annotated data frame object was merged with the normalized data set, leading to a complete gene data table. The selection criteria of significantly changed gene expression were based on an expression fold difference higher than $|2|$.

2.9. DAVID. Functional annotation clustering of differentially expressed genes was performed using DAVID (Database for Annotation, Visualization, and Integrated Discovery) [10]. Gene symbols for up- or downregulated genes from each of the compared groups were loaded into DAVID using the "RDAVIDWebService" BioConductor package. Functional annotation chats generated by DAVID with overrepresented gene annotations are shown as bubble plots from the BACA BioConductor package (https://cran.r-project. org/web/packages/BACA/BACA.pdf). Bubble plots were generated with the following criteria: $p$ value $<0.5$, adjusted method $=$ Benjamini, and minimal number of genes per group $=5$. Groups of genes fulfilling the mentioned criteria are presented in a graph in which the bubble size indicates the number of genes represented in the corresponding annotation and the condition of these genes in terms of their down- and upregulation.

2.10. Gene Set Enrichment Analysis (GSEA). GSEA is a computational method that is used for testing a priori-defined gene sets (GO terms, pathways) for their association with one of the two compared biological groups. The method utilizes the Kolmogorov-Smirnov (K-S) statistical test to identify significantly enriched or depleted groups of genes [11]. GSEA analysis has been conducted using the GSEA Java Desktop Application from the Broad Institute (http://software.broadinstitute.org/gsea/index.jsp). Normalized data from all genes were transformed into an appropriate format and imported into the application. Then, a predefined gene set database was selected from the Molecular Signatures Database (MsigDB) [12]. Genes belonging to the selected set were ranked according to the difference in their expression level using the signal-to-noise ratio with 1000 permutations. By walking down the ranked list of genes, the enrichment score (ES) was calculated for each selected gene set. This procedure was performed using a sum statistic when a gene was present in the gene set, and the calculation was decreased when it was not [12]. Enrichment scores were normalized by their gene set size, and false-positive findings were corrected by FDR. Significant gene sets were considered to be those with an adjusted nominal $p$ value $<0.01$ and FRD $q$ value $<25 \%$.

2.11. Statistical Methods. The significance of the differences between experimental groups was assessed by the MannWhitney $U$ test. $p<0.05$ was considered statistically significant. The data are presented as the mean \pm standard deviation (SD). Benjamini and Hochberg multiple testing correction was applied to correct for false-positive results in the microarray analysis.

\section{Results}

3.1. Functional and Morphological Evaluation of Aged Retinas from C3-I- and WT Mice. To assess whether complement component C3 deficiency influenced the physiological function of aged retinas, we examined the retinal bioelectrical response in 3- and 12-month-old C3-deficient (C3-/-) and control (wild type (WT)) mice. The results are summarized in Figure 1(a). The ERG recordings, under both scotopic and photopic conditions, in C3-/- and WT mice at 12 months of age, were significantly lower $(p<0.01)$ than those observed in 3-month-old gender-matched animals. When comparing bioelectrical responses of the 12-monthold C3-deficient and WT mice, we found considerable differences in b-wave amplitudes between the groups of animals. In $\mathrm{C} 3-/-$ mice, the mixed rod-cone response was significantly higher $(p<0.01)$ compared with that of the agematched controls $(72.81 \pm 21.13 \%$ versus $54.78 \pm 20.11 \%$, resp., in reference to b-wave amplitude measurements 

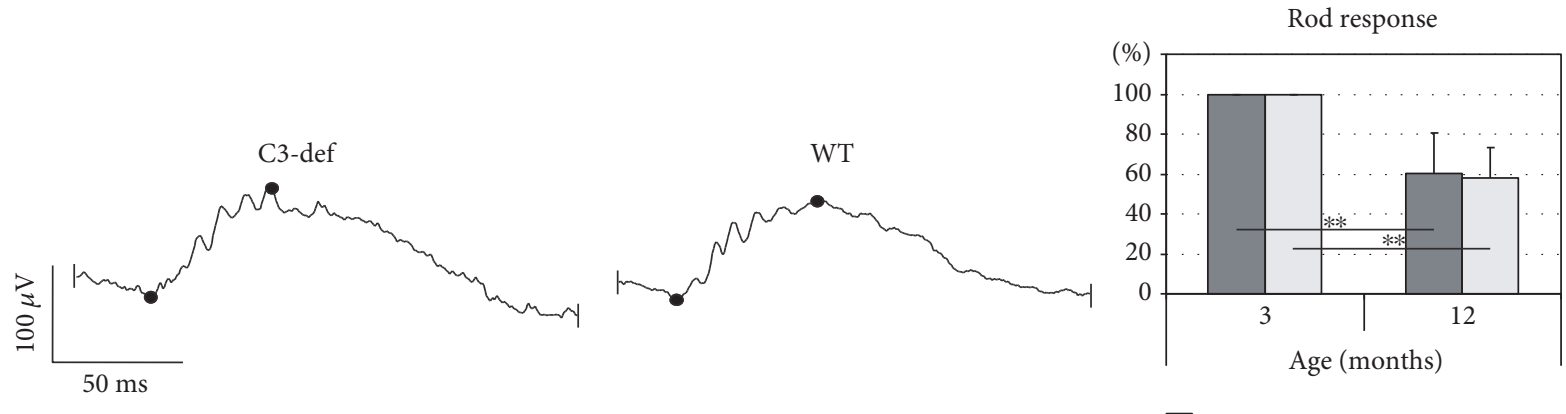

$\square$ C3-def

$\square$ WT

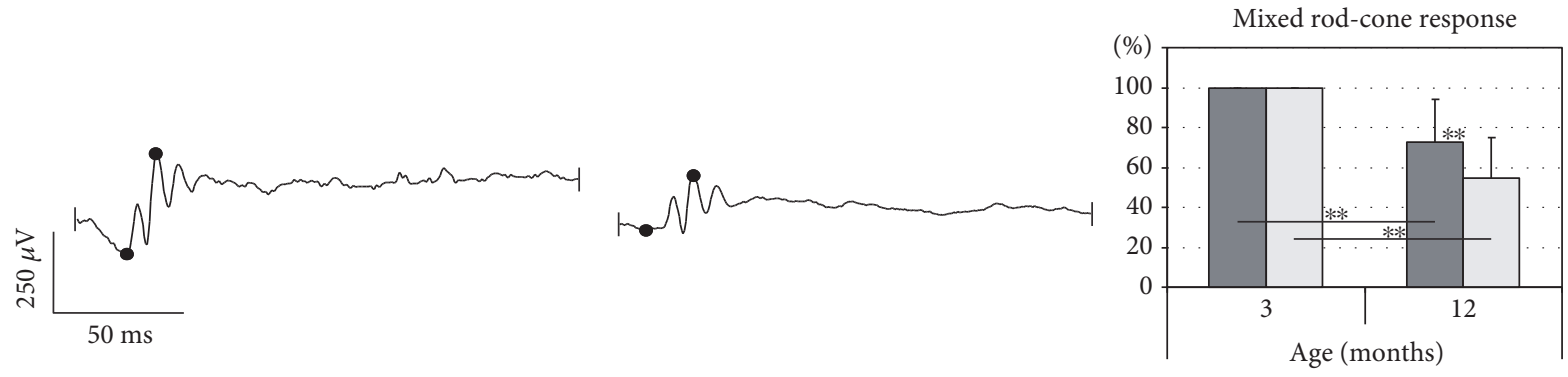

$\square$ C3-def

$\square$ WT

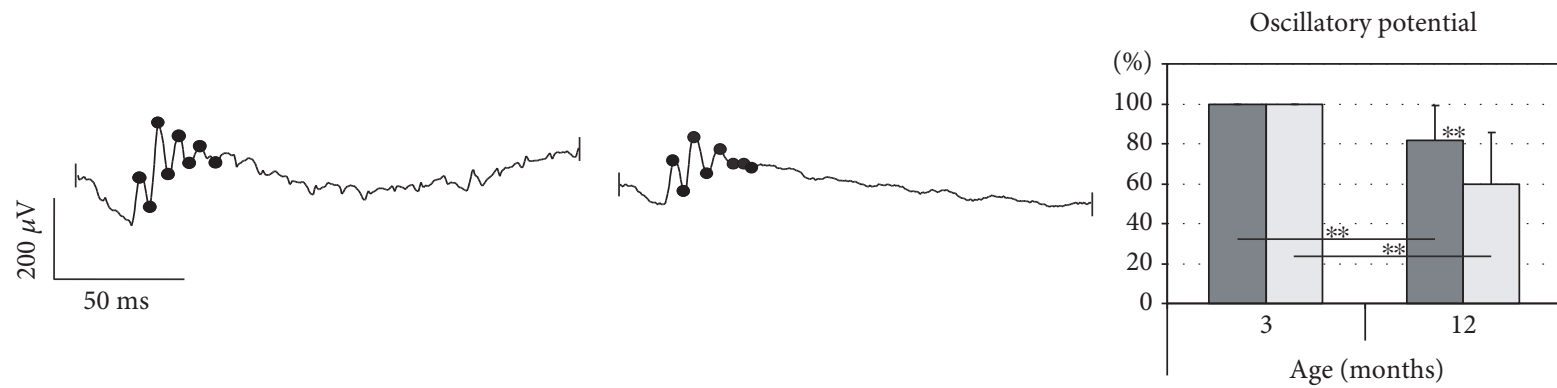

$\square$ C3-def

$\square$ WT

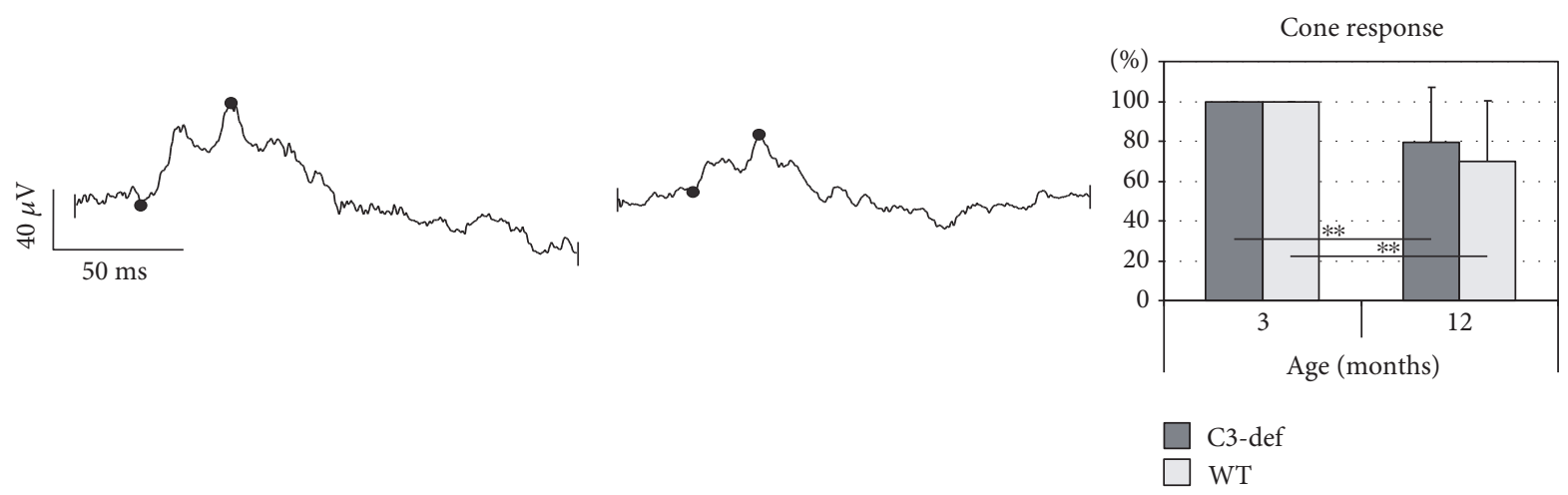

(a)

Figure 1: Continued. 
C3-def
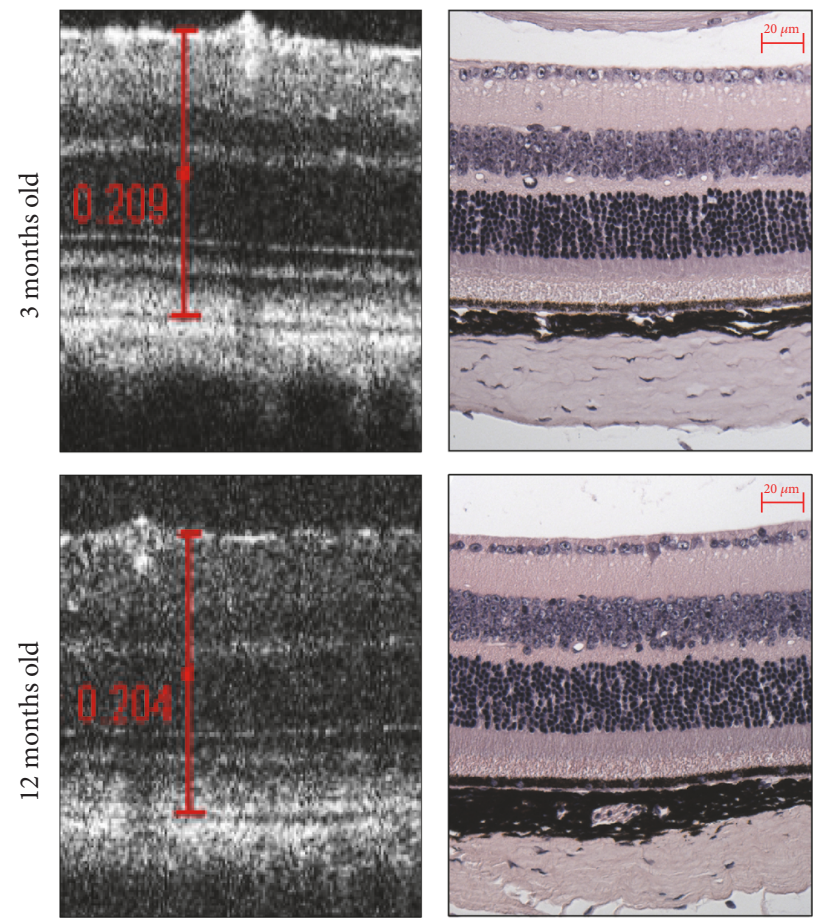

WT
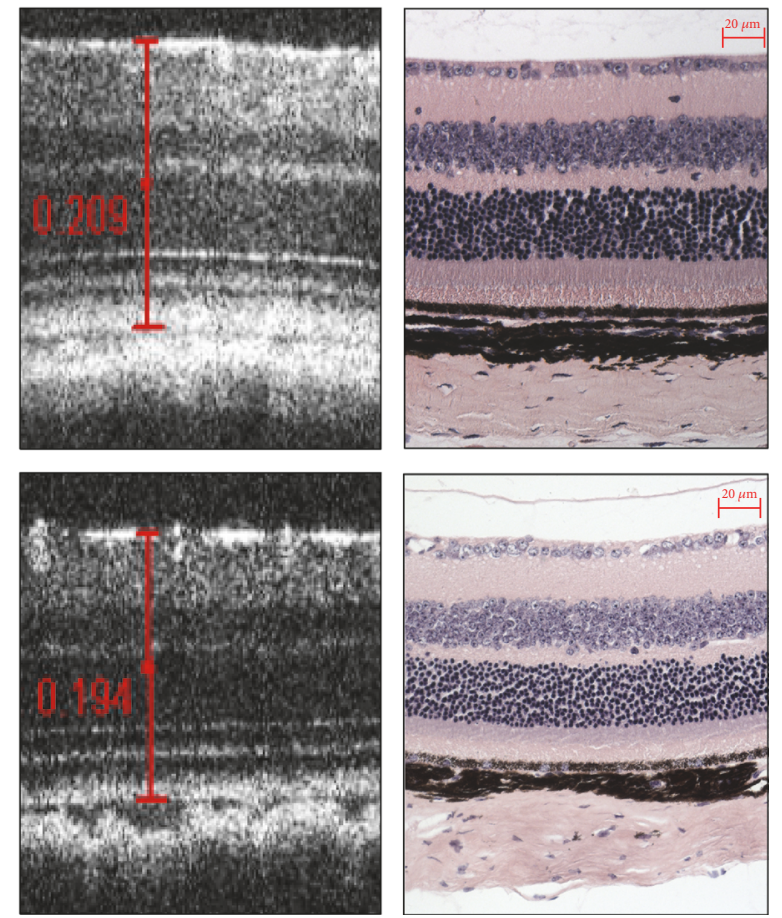

(b)

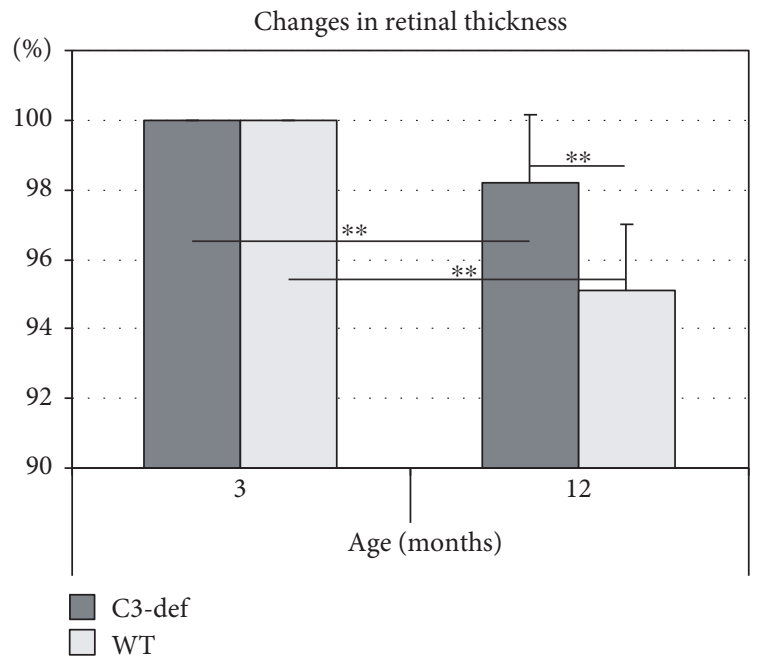

C3-def
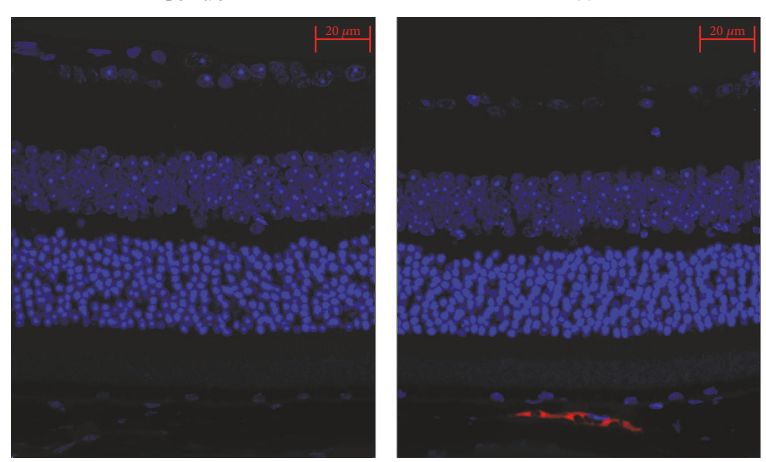

(c)

(d)

FIGURE 1: Functional and morphological evaluation of aged retinas from C3-/- and WT mice. (a) The representative rod, mixed rod-cone, and cone responses, as well as oscillatory potentials (OPs), recorded from 12-month-old C3-deficient (C3-def) and WT mice are shown. Changes in the retinal bioelectrical response during aging are presented as a percentage value calculated for each individual 1-year-old mice in reference to the b-wave amplitude measurements obtained from 3-month-old mice (considered 100\%). The results are shown as mean $\pm \mathrm{SD}$ ( $n=10$ mice per group). The ERG recordings at 12 months of age were reduced in both C3-/- and WT mice ${ }^{* *} p<0.01$ for scotopic and photopic conditions); however, when analyzing mixed rod-cone responses and OPs, the b-wave amplitudes in C3-deficient mice were significantly higher $\left({ }^{* *} p<0.01\right)$ compared to age-matched WT animals. (b) The representative in vivo SD-OCT retinal scans and H\&E-stained images of 3- and 12-month-old C3-def and WT mice are shown. The scale bar is $20 \mu \mathrm{m}$. (c) Spectral domain optical coherence tomography was used to assess the changes in retinal morphology and thickness over time. Changes in retinal thickness are presented as a percentage value calculated for each individual 1-year-old mice in reference to measurements obtained from 3-month-old mice (considered $100 \%)$. The results are shown as mean \pm SD ( $n=10$ mice per group). Aged retinas of both C3-def and WT mice showed the signs of declined thickness $\left({ }^{* *} p<0.01\right)$; however, this process was more profound in WT mice $\left({ }^{* *} p<0.01\right)$. (d) Immunofluorescence analysis of complement component C3 in aged WT retinas revealed deposition of the protein in the sub-RPE region. The sections from 12 -month-old C3-deficient animals served as a staining control. The scale bar is $20 \mu \mathrm{m}$. 
obtained from 3-month-old gender-matched mice, mean $\pm \mathrm{SD}, n=10$ mice per group). Similarly, the oscillatory potentials, which are thought to reflect the bioactivity initiated by amacrine cells in the inner retina, were considerably higher in C3-/- than in WT mice $(81.54 \pm 17.54 \%$ and $59.63 \pm 26.04 \%$, resp., $p<0.01)$. The differences of rod- and cone-driven response between $\mathrm{C} 3-1-$ and $\mathrm{WT}$ animals at 12 months of age were not statistically significant. These results may suggest that C3 deficiency affects the function of photoreceptors.

Consequently, to evaluate age-related changes in retinal morphology and thickness, we performed spectral domain optical coherence tomography (SD-OCT) in 12-month-old C3-deficient and WT mice. The results are summarized in Figures 1 (b) and 1(c). In both C3-/- and WT mice, the retinas at 12 months of age were thinner than those in the same mice at 3 months of age. When comparing the retinal thickness of the 12-month-old mice in both groups, the retinas of C3-deficient mice were found to be significantly $(p<0.01)$ thicker than those of the age-matched WT controls. These data provide evidence that the aged retinas of both C3-/- and WT mice exhibit age-related signs of reduced thickness; however, this process was significantly slower in C3-deficient mice.

To examine complement activation in aged WT mouse retinas, the immunofluorescence analysis of component C3 was applied. The sections from 12-month-old C3-deficient animals served as a staining control. We found depositions of $\mathrm{C} 3$ protein in the sub-RPE region (Figure $1(\mathrm{~d})$ ). This result suggests that complement activation may play a role in the process of natural retinal aging.

3.2. Oxidative Stress and Apoptosis Analysis in Aged Retinas from C3-I- and WT Mice. To determine a link between oxidative stress and the complement system, we performed expression analysis of selected proteins, including $4 \mathrm{HNE}$ (cellular oxidative stress marker), catalase (CAT), and glutathione reductase (GSR), in retinas collected from 12-monthold C3-/- mice and their WT counterparts. The results are summarized in Figure $2(\mathrm{a})$. We found that the $4 \mathrm{HNE} / \beta$-actin ratio was significantly lower in retinas collected from C3-/mice ( $n=14$ eyes per group, $p<0.01$ ). Moreover, CAT and GSR, which represent antioxidative enzymes, were expressed at significantly higher levels $(p<0.01)$ in $\mathrm{C} 3-/-$ mice. The above results suggest that aged retinas from old $\mathrm{C} 3-/-$ mice possess higher antioxidant enzyme activities than do the retinas of their age-matched counterparts and thus are less susceptible to the local development of oxidative stress in retinal tissue.

Next, to assess apoptosis as cell death-related processes, the Luminex multiplex fluorescent bead-based immunoassay was used to evaluate the expression levels of selected proteins associated with pro- and antiapoptotic cell signaling, including survivin, Mcl-1/Bak dimer, and the active form of caspase-3 in retinal lysates from both 12-month-old C3-/and WT mice $(n=14$ eyes per group). As shown in Figure 2(b), the C3-/- mice demonstrated higher expression levels of survivin $(p<0.01)$ and Mcl-1/Bak dimer $(p<0.05)$, which represent antiapoptotic proteins. In contrast, the expression of proapoptotic active caspase- 3 was significantly lower in $\mathrm{C} 3-/-$ mice $(p<0.05)$ than in WT mice.

3.3. Clearance of Modified or Misfolded Proteins in Aged Retinas from C3-/- and WT Mice. To examine the efficiency of age-related protein degradation mechanisms in retinal cells, we analyzed the expression of selected proteins involved in ubiquitination and autophagy, such as ubiquitin, LC3B-I, LC3B-II, and p62 from 12-month-old C3-/- and WT mice. The results are summarized in Figure 3. Since the conversion of LC3B-I to LC3B-II correlates well with the number of autophagosomes, the ratio of LC3B-II to LC3B-I was used as an indicator of autophagic activity. Accordingly, ubiquitin-binding p62 protein serves as a marker for the cargo subjected to autophagosomal degradation. As presented in Figure 3(a), we documented a significantly higher LC3B-II/I ratio $(p<0.05)$ together with a decreased expression of p62 protein $(p<0.01)$ in C3-deficient mice compared with the age-matched controls. Indeed, the Western blot analysis detected stronger bands of ubiquitin-tagged proteins in retinas collected from aged C3-/ - mice $(n=14$ eyes per group). Immunofluorescence staining of collected retinas confirmed the localization of ubiquitinated protein conjugates in the RPE layer (Figure 3(b)). Taken together, these observations imply that a complement system deficiency induces the cellular clearance of proteins through autophagy mechanisms in aged retinas.

3.4. Differential Gene Expression Profile. To identify molecular changes that are potentially responsible for the phenotype associated with the absence of complement component C3 in aged retina, we performed a detailed comparison of the transcriptional profiles of retinas isolated from 12-month-old C3-knockout and WT mice. Quantitative microarray analysis of RNA isolated from the collected retinas allowed the identification of 292 gene transcripts that showed at least a 2-fold significant difference in expression levels between the analyzed groups. Among them, 115 genes were overexpressed between 2- and 8.13-fold, whereas 177 genes were downregulated in a range from 2- to 25.95-fold in C3-1mice compared with the age-matched WT counterparts. The top 25 up- and downregulated genes in aged C3deficient mice are listed in Table 1. Within the group of overexpressed genes, the highest level of transcriptional activation was shown by enzymes that ensure vascular integrity, that is, serpin peptidase inhibitor and clade E (Serpine3), and the detoxification of metabolic products or environmental pollutants, that is, aldo-keto reductase family-1, member E1 (Akrle1). Moreover, C3-knockout mice demonstrated higher expression levels of genes involved in maintaining the physiological function of the neural retina: abhydrolase domain containing 14A (Abhd14a); complexin 4 (Cplx4); cGMP specific-phosphodiesterase 6C (Pde6c); neurofilament (Nefl); aldehyde dehydrogenase family 1, subfamily A1 (Aldh1a1); retinitis pigmentosa GTPase regulator interacting protein-1 (Rpgrip1); and regulator of $\mathrm{G}$ protein signaling 9 binding protein $(R g s 9 b p)$. The most downregulated genes in retinas from C3-deficient mice were those associated with innate and adaptive 

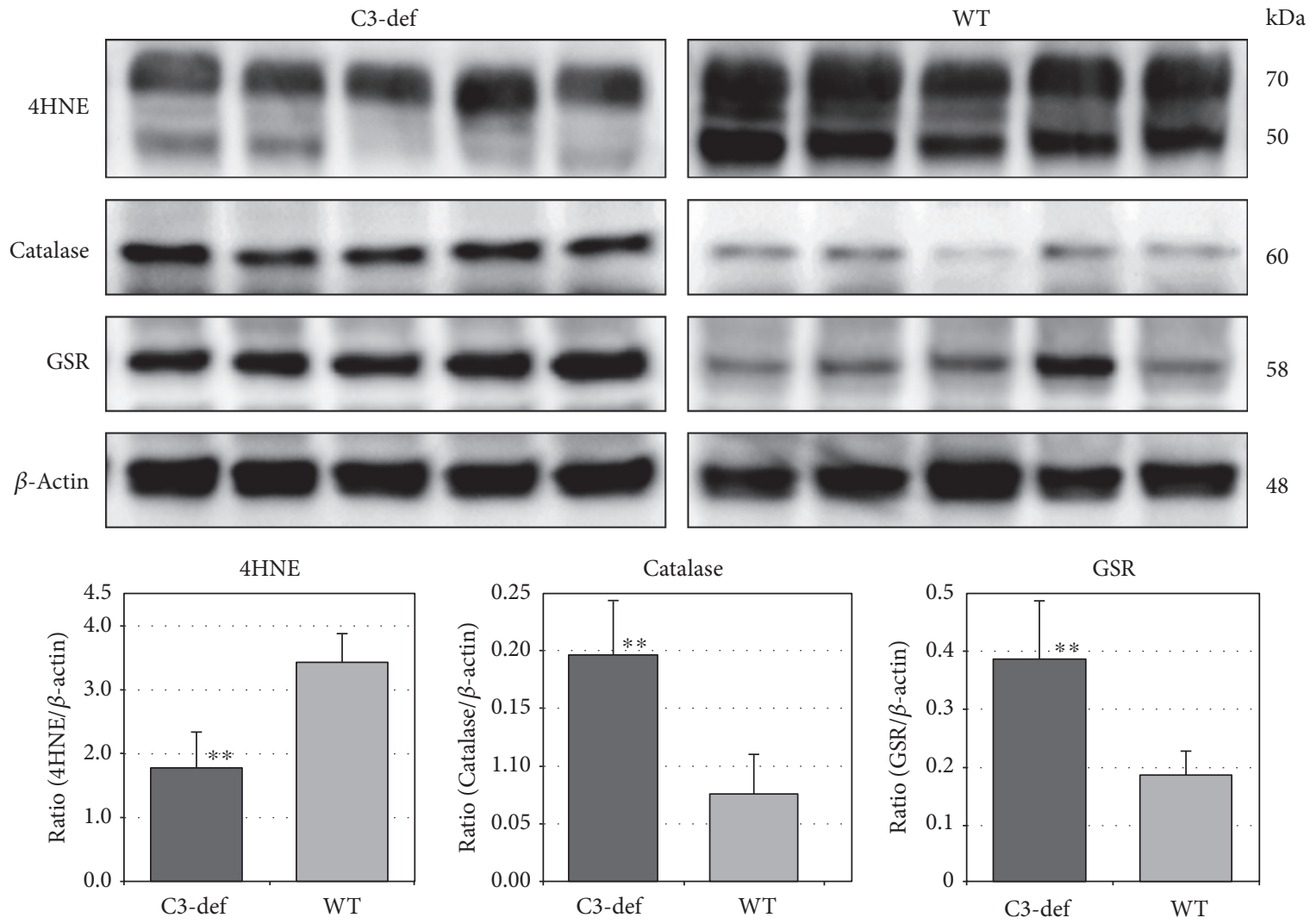

(a)
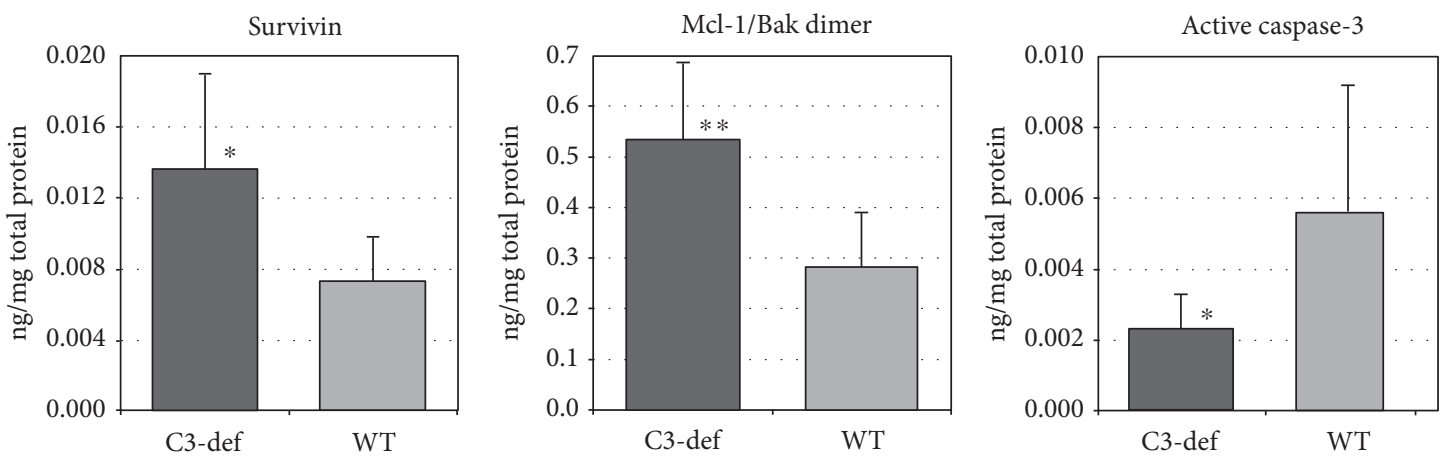

(b)

FIGURE 2: Evaluation of oxidative stress markers and apoptotic activity in the retinas of aged C3-deficient and WT mice. (a) Western blot and densitometry analysis of $4 \mathrm{HNE}$, catalase, glutathione reductase (GSR), and $\beta$-actin, which served as an internal control. Immunoblot images are representative of three independent experiments yielding similar results. The protein expression level of the $4 \mathrm{HNE}$, catalase, and GSR was quantified in comparison to that of $\beta$-actin and shown as mean \pm SD ( $n=14$ eyes per group). $4 \mathrm{HNE}$ modified proteins, an oxidative stress marker, were detected in both 12-month-old C3-def and WT mice, although the 4HNE/ $\beta$ actin ratio was significantly lower in the retinas collected from C3-def mice $\left({ }^{* *} p<0.01\right)$. Moreover, catalase and GSR levels, which represent antioxidant enzymes, were considerably higher in C3-deficient mice $\left({ }^{* *} p<0.01\right)$. (b) Luminex multiplex fluorescent bead-based immunoassay was used to evaluate protein expression level of survivin, Mcl-1/Bak dimer, and active caspase-3 in retinal lysates. To standardize the final concentration values, the obtained data was normalized to the total protein concentration. The results are shown as mean \pm SD ( $n=14$ eyes per group). C3-def mice demonstrated higher rates of survivin and Mcl-1/Bak dimer $\left({ }^{* *} p<0.01\right)$, which represent antiapoptotic proteins $\left({ }^{*} p<0.05\right)$. The rates of active caspase- 3 were considerably lower in C3-def mice $\left({ }^{*} p<0.05\right)$ as compared to agematched control mice.

immunity: regenerating islet-derived $3 \gamma(\operatorname{Reg} 3 g)$, proplatelet basic protein $(P p b p)$, defensin $\beta 6$ (Defb6), lactotransferrin $(L t f)$, mucin $5 \mathrm{~B}(M u c 5 b)$, and a large group of genes coding basic structural units of immunoglobulins-Ighj4,
Igkv4-53, Igh-VJ558, Ighj1, Igj, Igkj1, Ighg2b, Ighj3, Ighg1, and Igkv1-110.

Furthermore, up- and downregulated genes were assigned to specific biological processes according to the 

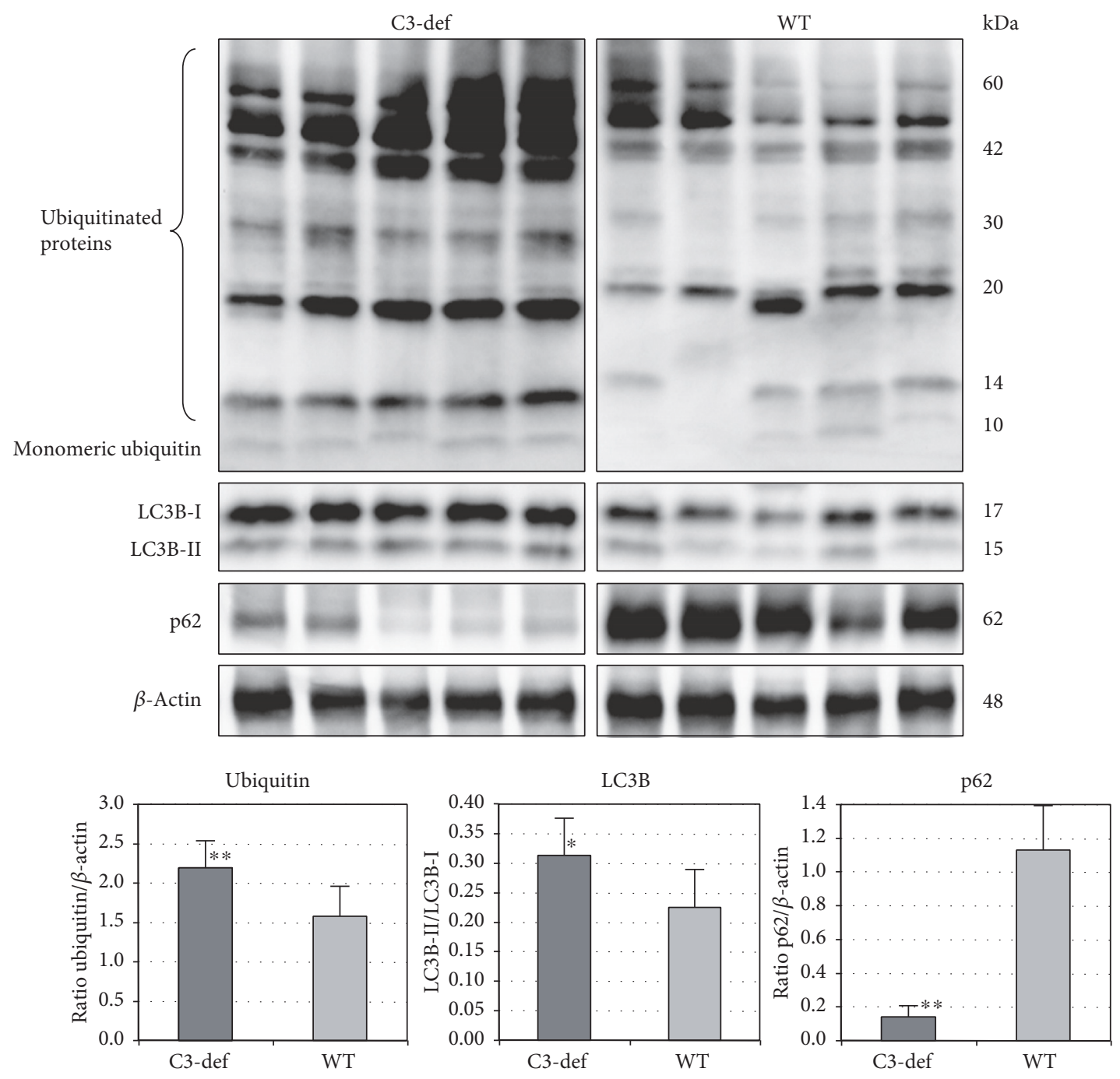

(a)

C3-def

WT
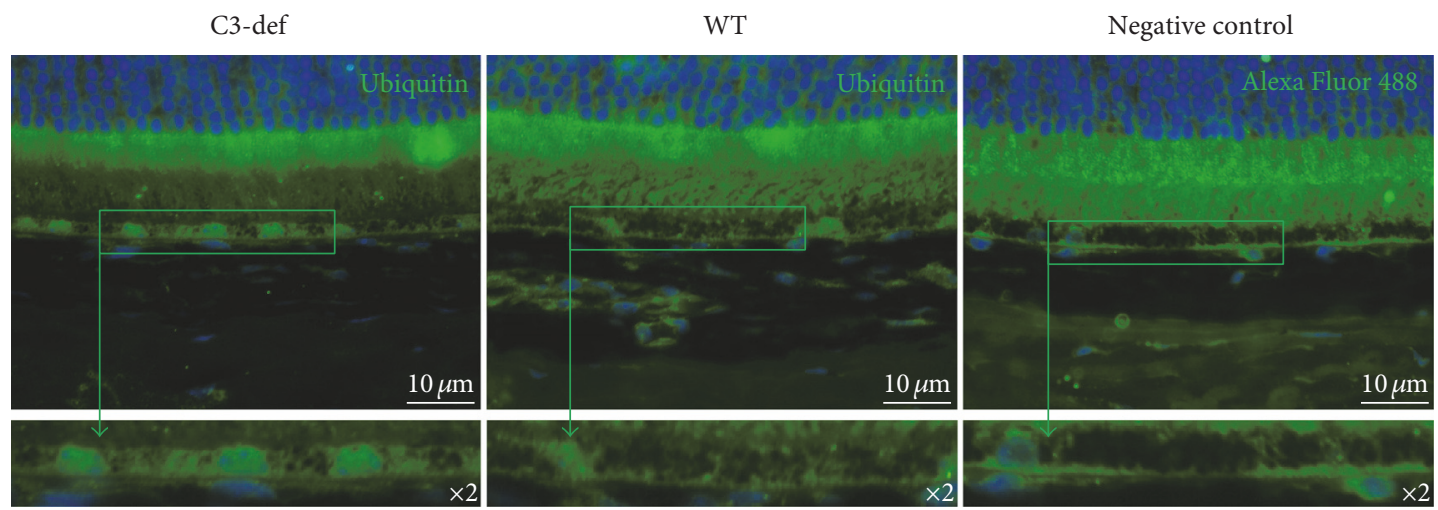

(b)

FIGURE 3: Evaluation of the autophagy markers in the retinas of aged C3-deficient and WT mice. (a) Western blot and densitometry analysis of ubiquitin, LC3B, p62, and $\beta$-actin, which served as an internal control. Immunoblot images are representative of three independent experiments yielding similar results. The protein expression level of the ubiquitin and $\mathrm{p} 62$ was quantified in comparison to that of $\beta$-actin. The LC3B protein level is presented as a LC3B-II/LC3B-I ratio. The results are shown as mean \pm SD ( $n=14$ eyes per group). Western blot analysis in the retinas collected from C3-def mice demonstrates strong expression of the ubiquitinated proteins $\left({ }^{* *} p<0.01\right)$ and higher LC3B-II/I ratio $\left({ }^{*} p<0.05\right)$ together with a decrease in p62 level $\left({ }^{* *} p<0.01\right)$, compared to that from age-matched control mouse retinas. (b) Immunofluorescence staining of ubiquitin protein in the retinas of the 12-month-old C3-def and WT mice confirms the decreased expression of ubiquitin in RPE cells of WT mice. The scale bar is $10 \mu \mathrm{m}$. 
TABLE 1: List of top 25 up- and downregulated genes in 12-month-old C3-deficient mice. Genes are ranked according to the expression fold difference-the gene from the top of the list is the most upregulated and the gene from the bottom of the list is the most downregulated in C3-deficient mice compared to control animals.

\begin{tabular}{|c|c|c|c|c|c|}
\hline Direction in C3-def & Gene symbol & Gene name & Probe ID & Entrez Gene ID & Fold change \\
\hline Up & Serpine3 & $\begin{array}{c}\text { Serpin peptidase inhibitor, clade E (nexin, plasminogen } \\
\text { inhibitor) }\end{array}$ & 17301155 & 319433 & 8.13 \\
\hline Up & Akrle1 & Aldo-keto reductase family 1, member E1 & 17290301 & 56043 & 4.41 \\
\hline Up & Edv & $\begin{array}{l}\text { Endogenous sequence related to the Duplan murine } \\
\text { retrovirus }\end{array}$ & 17548311 & 13623 & 4.28 \\
\hline Up & Rpl29 & Ribosomal protein 29 & 17521204 & 19944 & 4.23 \\
\hline Up & Abhd14a & Abhydrolase domain containing 14A & 17530686 & 68644 & 4.02 \\
\hline Up & Gatc & $\begin{array}{l}\text { Glutamyl-tRNA (Gln) amidotransferase, subunit } \\
\text { C homolog }\end{array}$ & 17451721 & 384281 & 3.70 \\
\hline Up & Cplx4 & Complexin 4 & 17355086 & 225644 & 3.33 \\
\hline $\mathrm{Up}$ & Uprt & Uracil phosphoribosylotransferase (FUR1) homolog & 17537003 & 331487 & 3.07 \\
\hline $\mathrm{Up}$ & $\mathrm{H} 2-\mathrm{T} 22$ & Histocompatibility $2, \mathrm{~T}$ region locus 22 & 17344593 & 15039 & 3.02 \\
\hline Up & Mzt1 & Mitotic spindle organizing protein 1 & 17309065 & 76789 & 3.01 \\
\hline Up & Mpc1 & Mitochondrial pyruvate carrier 1 & 17284037 & 55951 & 2.81 \\
\hline Up & Adam4 & A disintegrin and metallopeptidase domain 4 & 17282343 & 11498 & 2.78 \\
\hline Up & Rab6b & RAB6B, member RAS oncogene family & 17520922 & 270192 & 2.75 \\
\hline Up & Pcdhb18 & Protocadherin beta 18 & 17349930 & 93889 & 2.67 \\
\hline Up & Cdh12 & Cadherin 12 & 17310550 & 215654 & 2.65 \\
\hline Up & Fabp4 & Fatty acid-binding protein 4 , adipocyte & 17404091 & 11770 & 2.59 \\
\hline Up & Pde6c & Phosphodiesterase 6C, cGMP specific, cone, alpha prime & 17359118 & 110855 & 2.59 \\
\hline Up & Fam213a & Family with sequence similarity 213 , member A & 17305221 & 70564 & 2.59 \\
\hline Up & Nefl & Neurofilament, light peptide & 17301582 & 18039 & 2.57 \\
\hline Up & Akap6 & A kinase (PRKA) anchor protein 6 & 17275436 & 238161 & 2.57 \\
\hline Up & Aldh1a1 & Aldehyde dehydrogenase family 1 , subfamily A1 & 17358103 & 11668 & 2.55 \\
\hline Up & Hdac1 & Histone deacytelase 1 & 17430397 & 433759 & 2.55 \\
\hline Up & Rpgrip1 & Retinitis pigmentosa Gtpase regulator-interacting protein 1 & 17299715 & 77945 & 2.54 \\
\hline Up & Rgs9bp & Regulator of $\mathrm{G}$ protein signaling 9 binding protein & 17489809 & 243923 & 2.50 \\
\hline Down & Il1f9 & Interleukin 1 family, member 9 & 17367652 & 215257 & -5.53 \\
\hline Down & Igkv1-110 & Immunoglobulin kappa variable $1-110$ & 17459338 & 381777 & -5.64 \\
\hline Down & Muc5b & Mucin 5, subtype B, tracheobronchial & 17485098 & 74180 & -6.06 \\
\hline Down & Krt10 & Keratin 10 & 17269064 & 16661 & -6.11 \\
\hline Down & Krtdap & Keratinocyte differentiation-associated protein & 17476557 & 64661 & -6.39 \\
\hline Down & Sprr2f & Small proline-rich protein $2 \mathrm{~F}$ & 17399876 & 20760 & -6.46 \\
\hline Down & Ighg1 & Immunoglobulin heavy constant gamma 1 (G1m marker) & 17284339 & 16017 & -6.48 \\
\hline Down & Ighj3 & Immunoglobulin heavy joining 3 & 17284356 & 777655 & -7.50 \\
\hline Down & Ighg $2 b$ & Immunoglobulin heavy constant gamma $2 \mathrm{~B}$ & 17284334 & 16016 & -7.73 \\
\hline Down & Igkj1 & Immunoglobulin kappa joining 1 & 17459415 & 110759 & -8.18 \\
\hline Down & Defb6 & Defensin beta 6 & 17499602 & 116746 & -8.34 \\
\hline Down & Igj & Immunoglobulin joining chain & 17449447 & 16069 & -8.53 \\
\hline Down & Cryba4 & Crystalline, beta A4 & 17451195 & 12959 & -8.55 \\
\hline Down & Ltf & Lactotransferrin & 17522555 & 17002 & -8.70 \\
\hline Down & Cnfn & Cornifelin & 17487919 & 72383 & -8.97 \\
\hline Down & Ighj1 & Immunoglobulin heavy joining 1 & 17284360 & 777648 & -10.53 \\
\hline Down & Klk13 & Kallikrein-related peptidase 13 & 17477191 & 626834 & -10.71 \\
\hline Down & Lgsn & Lengsin, lens protein with glutamate synthetase domain & 17211533 & 266744 & -11.56 \\
\hline Down & Lcelc & Late cornified envelope $1 \mathrm{C}$ & 17399899 & 73719 & -12.31 \\
\hline Down & Igh-VJ558 & Immunoglobulin heavy chain (J558 family) & 17284314 & 16061 & -13.62 \\
\hline
\end{tabular}


TABLe 1: Continued.

\begin{tabular}{|c|c|c|c|c|c|}
\hline Direction in C3-def & Gene symbol & Gene name & Probe ID & Entrez Gene ID & Fold change \\
\hline Down & Rps13 & Ribosomal protein S13 & 17493182 & 68052 & -14.76 \\
\hline Down & Igkv4-53 & Immunoglobulin kappa variable 4-53 & 17459423 & 546213 & -16.50 \\
\hline Down & Ighj4 & Immunoglobulin heavy joining 4 & 17284354 & 777656 & -18.58 \\
\hline Down & Ppbp & Proplatelet basic protein & 17438963 & 57349 & -21.02 \\
\hline Down & Reg3g & Regenerating islet-derived 3 gamma & 17467973 & 19695 & -25.95 \\
\hline
\end{tabular}

Gene Ontology (GO) classification. The bubble diagram illustrating overrepresented terms is shown in Figure 4. Analysis of functional annotations identified two biological processes that were significantly upregulated in C3knockout animals: visual perception and sensory perception of light stimulus. These processes are represented in our study by several upregulated genes: Gucy2f, Ppt1, Bbs2, Crb1, Gabrr2, Rgs9bp, Rpgrip1, and Pde6c, which are essential for photoreceptor-based light-dependent signal transformation and transduction. In contrast, among the biological processes that were inhibited in C3-/- mice were those such as the immune response, defense response, humoral immune response, humoral immune response mediated by circulating immunoglobulin, and immune system process regulation. Further analysis of the GO functional annotations also revealed other general terms that were of interest. It should be emphasized that the GO database is still in the development stage and is far from being complete. Moreover, it is composed of some general as well as specific categories with a similar meaning; therefore, a single gene may be mapped to several GO terms and may be counted more than once [13].

To more closely examine the biological influence of $\mathrm{C} 3$ deficiency on the aged retina, we performed gene set enrichment analysis (GSEA) to identify pathways that were altered in 12-month-old C3-/- mice compared with WT animals. GSEA detected 26 gene sets that were enriched in mouse knockouts for the $\mathrm{C} 3$ gene. Most of these were involved in neurological processes, such as the secretory pathway (normalized enrichment score $(\mathrm{NES})=2.02$ ), synaptic transmission (1.98), transmission of nerve impulses (1.96), neurological system processes (1.90), synaptogenesis (1.75), phototransduction (1.70), nervous system development (1.67), synapse organization and biogenesis (1.66), central nervous system development (1.62), and sensory perception (1.61). Simultaneously, 29 gene sets were negatively correlated to C3-/- mice. Among them, the two largest groups were represented by gene sets associated with the immune system: response to bacterium $(-2.12)$, defense response $(-1.98)$, regulation of the immune response $(-1.76)$, regulation of immune system processes (-1.68), and immune system processes $(-1.61)$, and inflammation: acute inflammatory response $(-2.05)$, inflammatory response $(-1.76)$, cytokine- and chemokine-mediated signaling pathway $(-1.73)$. Representative diagrams of the enriched gene sets are shown in Figure 5. A complete list of gene sets enriched in both aged C3-/- and WT mice is presented in Table 2.

\section{Discussion}

There is a general consensus that cumulative oxidative damage is responsible for aging and may therefore play an important role in the pathogenesis of retinal degeneration [14]. The retina is constantly challenged by excessive formation of ROS due to the high metabolic rate (i), high oxygen consumption (ii), long periods of exposure to short-wavelength light (iii), high concentration of polyunsaturated fatty acids (PUFAs) (iv), and phagocytic activity of RPE cells, which is accompanied by a respiratory burst-a rapid release of superoxide radical and hydrogen peroxide (v) [14-16]. Several studies have documented that the expression of intrinsic antioxidants, including catalase and glutathione reductase, has been shown to decline with age [17-19] and in AMD patients $[17,20]$. In our study, we found that 12-month-old C3-/- mice exhibited lower levels of 4HNE-modified proteins-a marker of oxidative damage-compared with age-matched WT mice. Moreover, the expression of two antioxidant enzymes, catalase and glutathione reductase, was significantly increased in aged C3-/- mice. Our findings suggest that CS may act as a mediator of oxidative stress and local tissue injury. Indeed, a number of studies suggest that prolonged oxidative stress may cause retinal damage through a local dysregulation of the complement system [21]. Human ARPE-19 cells exposed to low-grade oxidative stress exhibited markedly impaired secretion of several complement regulators, including membrane-bound (DAF, MCP, and CD59) and fluid-phase (CFH) molecules [22-24], leading to uncontrolled complement activation.

It has also been proposed that protein modification by $4 \mathrm{HNE}$ is an early event that precedes photoreceptor cell apoptosis [25]. Herein, we found that the level of active caspase3 , a hallmark of the ongoing irreversible phase of apoptosis, was significantly lower in retinas from aged C3-/- mice compared with control animals and corresponded to significantly lower levels of $4 \mathrm{HNE}$ in retinas from old C3-/- mice. In addition, the levels of two other antiapoptotic proteins, survivin and Mcl-1/Bak dimer, were upregulated in C3deficient animals. We infer that in C3-/- mice, the naturally lower levels of oxidative stress may explain the decreased age-related thinning of the retina observed in these animals as determined by OCT. Furthermore, C3-knockout mice appeared to be potentially protected from apoptosis by generating higher levels of antiapoptotic proteins such as survivin and $\mathrm{Mcl}-1 / \mathrm{Bak}$ dimer.

In an attempt to characterize the mechanisms underlying the beneficial effects of $\mathrm{C} 3$ deficiency, we analyzed the activity 


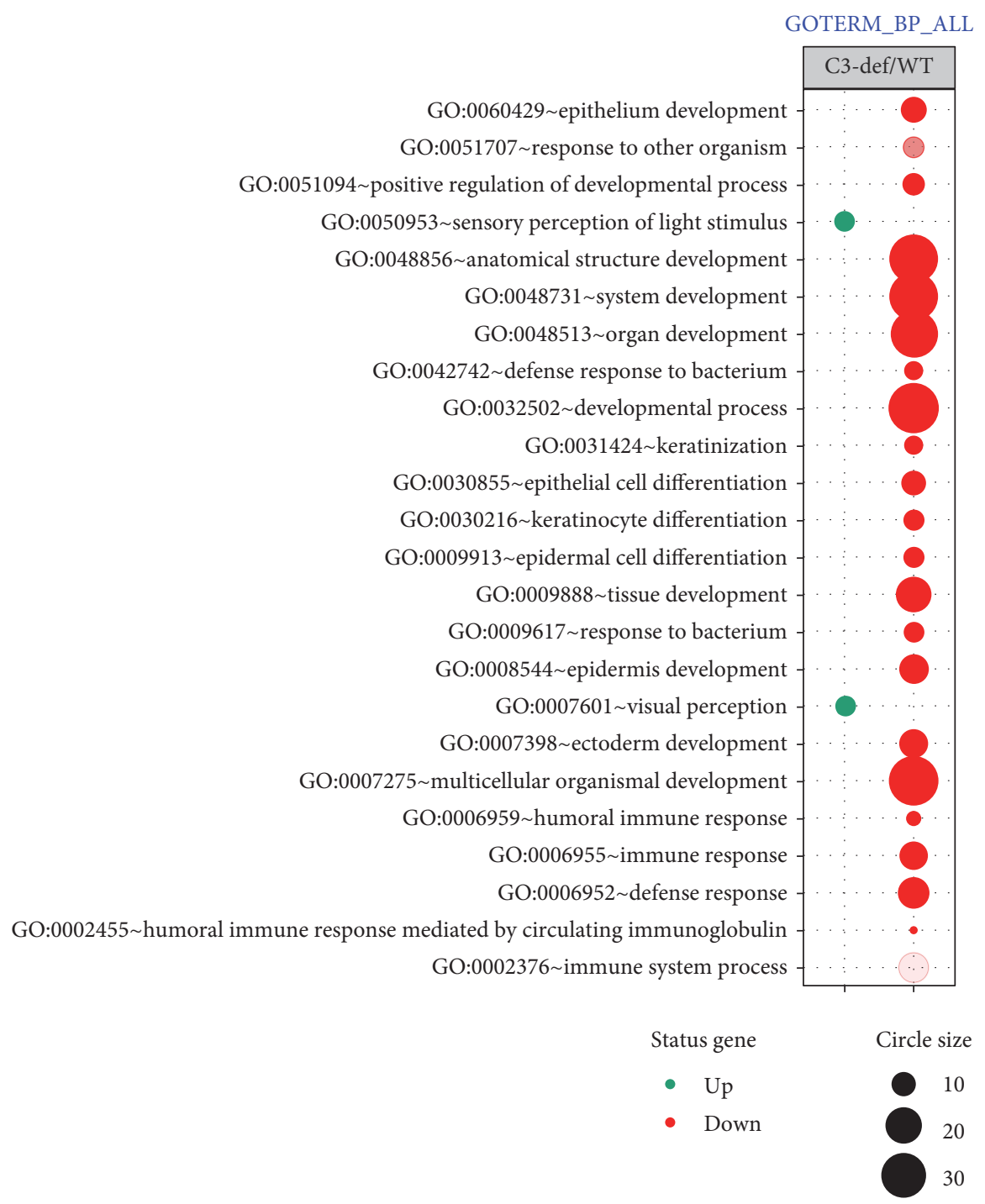

FIGURE 4: Overrepresented biological process assigned according to Gene Ontology (GO) classification in the retinas of aged C3-deficient mice compared to WT mice. Groups of genes fulfilling criteria: adjusted $p$ value $<0.05$, method $=$ Benjamini, and minimum number of genes per group $=5$, are presented in a graph, where bubble size indicates the number of genes represented in corresponding annotation and the condition of these genes in terms of their up- and downregulation.

of autophagy and proteasomal cleansing in retinal cells and found that the absence of active C3 had a significant effect on the quality of this process. It is widely accepted that dysregulation of the UPP and autophagy processes is associated with a detrimental accumulation of aggregated proteins, which further increases the oxidative burden and can lead to the development of retinal degenerative disorders [4, 26, 27]. The UPP system is responsible for the degradation of $80 \%-90 \%$ of proteins, mostly short-lived, oxidatively modified, or damaged proteins [28, 29]. Autophagy is specialized to degrade aggregated proteins and cellular organelles [30]. The UPP and autophagy were previously regarded as two independent processes; however, it has recently been suggested that both cooperate with each other because ubiquitinylation may target substrates for degradation via both pathways $[30,31]$. It has been documented that abundant oxidative stress negatively affects the autophagy process. Current data indicate that Nrf2 transcription factor, which is a key regulator of the oxidative stress defense system, interacts closely with the autophagy process via p62 protein [32-34]. Nrf2-knockout mice developed age-dependent degeneration of the RPE and choriocapillaries, spontaneous neovascularization, and deposits of inflammatory proteins in the sub-RPE space due to impaired autophagy and proteasomal cleansing processes. Moreover, in the RPE and Bruch's membrane of Nrf2-deficient mice, an age-dependent increase in immunoreactivity of C3d (a marker of complement activation), vitronectin (complement S-protein), and 3-nitrotyrosine (a marker of oxidatively damaged proteins) was observed [32]. The ubiquitin-binding p62/SQSTM1 (p62) protein serves as a marker for the cargo that is subjected to autophagosomal degradation [35]. Classically, the p62 level is expected to decrease due to degradation during the active autophagic 

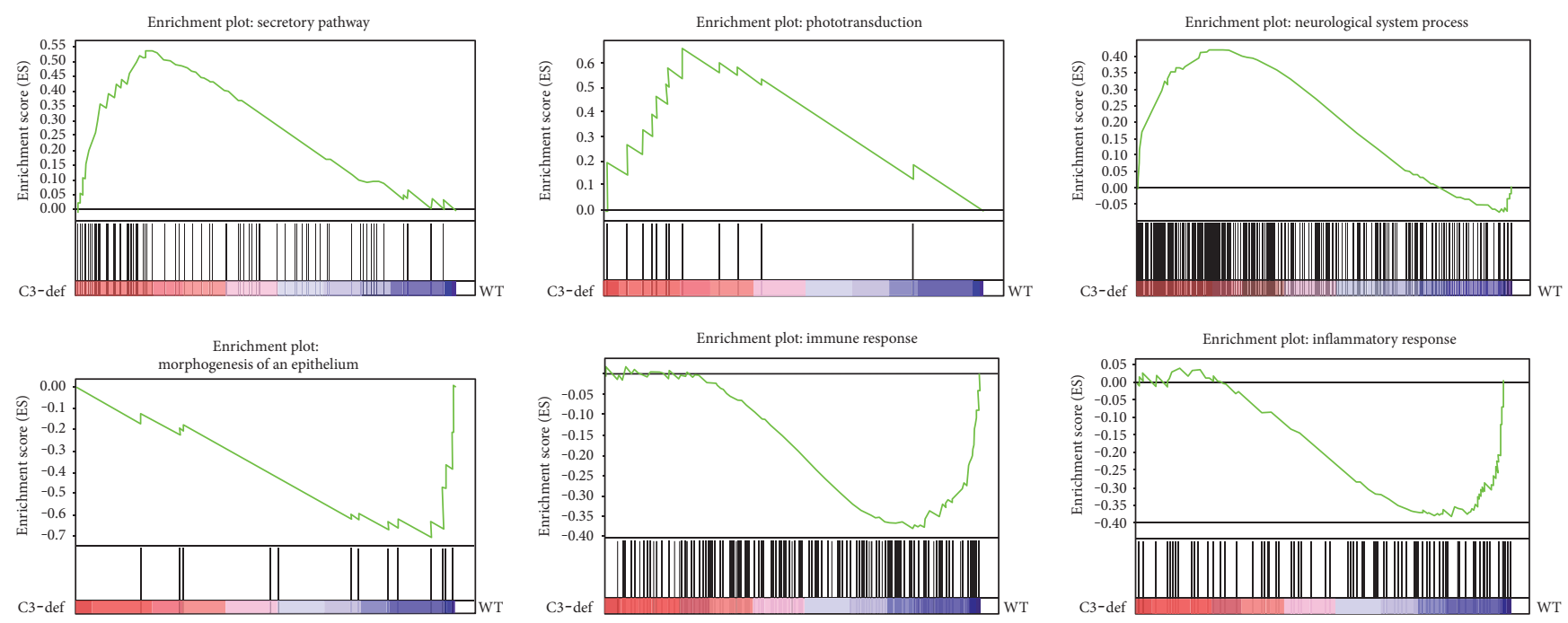

FIGURE 5: Gene set enrichment analysis (GSEA) of the retinas from aged C3-deficient mice compared to WT mice. Diagrams represent selected gene sets enriched in C3-deficient mice (secretory pathway, phototransduction, and neurological system process) and WT control animals (morphogenesis of an epithelium, immune response, and inflammatory response).

process, and the increased expression of p62 indicates impaired autophagosomal degradation [36]. Indeed, retina samples from AMD patients showed an accumulation of p62 [37]. Another protein that is crucial for autophagy, LC3B-I, is found in preautophagosomal structures and converted to the LC3B-II form during the autophagosome maturation process. Finally, the mature autophagosomes are degraded by lysosomal hydrolytic enzymes [38]. Our group demonstrated a reduced expression of p62 and an elevated expression of the LC3B-II/LC3B-I ratio in retinas from aged C3-/- compared with WT mice. This result may indicate the good quality of the ongoing process of autophagosome formation in $\mathrm{C} 3-/-$ mice and the successful packaging of waste material for autophagic degradation. The use of anti-ubiquitin antibody to monitor the localization of ubiquitin-positive cells further confirmed the Western blotting results. The ubiquitinated protein aggregate signal was higher in aged C3-l- retinal samples than in the WT controls and was mainly localized in the RPE layer. This finding reveals that C3 deficiency increases the amount of aggregate-prone proteins that undergo autophagy cleansing. Overall, our results indicate that CS suppression might facilitate the intracellular autophagic flux, as evidenced by the low levels of p62 together with high LC3B-II in the aged retinas.

Our findings support the notion that the ameliorative effects of C3 deficiency result from the compensatory ability to modulate the expression of several genes and proteins, which are components of signal transduction pathways that are important for retinal cell survival and function. Likewise, we were able to detect profound changes in the global gene expression profile using a comprehensive RNA microarray. Within the group of overexpressed genes in aged C3-/- animals, the highest levels of transcriptional activation were shown by Serpine3 (8.13-fold), Akrle1 (4.41-fold), Cplx4 (3.33-fold), Pde6c (2.59-fold), Rpgrip (2.54-fold), and Rgs $9 \mathrm{bp}$ $(2.50$-fold). The Serpine3 gene encodes an extracellular protein that belongs to the protease inhibitor family with activity toward thrombin, trypsin, and urokinase. It promotes neurite extension by inhibiting thrombin, and it possesses antiangiogenic properties [39]. Akrlel is a member of the aldo-keto reductase family, which has cytoprotective effects against oxidative stress $[40,41]$. Complexin 4 (Cplx4) is expressed mainly in photoreceptor and bipolar cell synapses [42]. Members of this protein family are responsible for the regulation of vesicle fusion and transmitter release, thus acting as a mediator of visual signal transmission [43]. Pde6c and Rgs $9 b p$ are both associated with normal phototransduction processes in cones and rods, respectively [44]. Retinitis pigmentosa-GTPase regulator-interacting protein (Rpgrip) has an important role in photoreceptor connecting cilia, helping to maintain correct photoreceptor function [45]. Based on the Gene Ontology classification of the biological processes, we identified two pathways that were significantly upregulated in aged C3-knockout mice: visual perception and sensory perception of light stimulus. Moreover, in addition to the results obtained using the GO database, GSEA detected 26 gene sets that were enriched in mice lacking the C3 gene, some of which were involved in neurological processes such as the secretory pathway, synaptic transmission, transmission of nerve impulses, neurological system processes, synaptogenesis, phototransduction, nervous system development, synapse organization and biogenesis, central nervous system development, and sensory perception. Of note, complement C3 naturally mediates immunerelated activities, but it also facilitates other processes such as synapse elimination in the developing visual system of young mice [46]. However, in the aged brain, C3 contributes to region-specific synapse and neuron loss [47].

Furthermore, since component C3 plays a potent role in the host defense system, the most downregulated genes in aged C3-deficient mice were those associated with innate and adaptive immunity. These results are consistent with previous reports that $\mathrm{C} 3$-deficient subjects are more 
TABLE 2: Top gene sets enriched in 12-month-old C3-deficient and WT mice. Gene sets are ranked according to the normalized enrichment score (NES) - the gene set from the top of the list is the most enriched in C3-def mice and the gene set from the bottom of the list is the most enriched in WT mice.

\begin{tabular}{|c|c|c|c|c|c|c|}
\hline Direction in C3-def & Gene set & Size & ES & NES & NOM $p$ value & FDR $q$ value \\
\hline Up & Secretory pathway & 83 & 0.54 & 2.02 & 0.000 & 0.014 \\
\hline Up & Synaptic transmission & 164 & 0.48 & 1.98 & 0.000 & 0.021 \\
\hline Up & Transmission of nerve impulse & 179 & 0.47 & 1.96 & 0.000 & 0.021 \\
\hline Up & Neurological system process & 359 & 0.42 & 1.90 & 0.000 & 0.038 \\
\hline Up & Nuclear export & 32 & 0.60 & 1.84 & 0.000 & 0.076 \\
\hline Up & Golgi vesicle transport & 48 & 0.54 & 1.81 & 0.000 & 0.097 \\
\hline Up & Brain development & 47 & 0.53 & 1.80 & 0.000 & 0.090 \\
\hline Up & Secretion by cell & 114 & 0.45 & 1.79 & 0.000 & 0.101 \\
\hline Up & Exocytosis & 24 & 0.60 & 1.76 & 0.007 & 0.126 \\
\hline Up & Synaptogenesis & 15 & 0.66 & 1.75 & 0.009 & 0.115 \\
\hline Up & RNA export from nucleus & 19 & 0.63 & 1.73 & 0.003 & 0.131 \\
\hline Up & Regulation of cell-cell adhesion & 9 & 0.77 & 1.72 & 0.007 & 0.135 \\
\hline Up & Microtubule-based movement & 16 & 0.64 & 1.71 & 0.007 & 0.150 \\
\hline Up & Phototransduction & 13 & 0.66 & 1.70 & 0.007 & 0.154 \\
\hline Up & Nervous system development & 352 & 0.37 & 1.67 & 0.000 & 0.187 \\
\hline Up & Chromosome organization and biogenesis & 118 & 0.42 & 1.67 & 0.000 & 0.184 \\
\hline Up & NLS-bearing substrate import into nucleus & 12 & 0.67 & 1.67 & 0.017 & 0.175 \\
\hline Up & Synapse organization and biogenesis & 20 & 0.60 & 1.66 & 0.022 & 0.174 \\
\hline Up & Establishment of cellular localization & 343 & 0.36 & 1.63 & 0.000 & 0.216 \\
\hline Up & Central nervous system development & 116 & 0.41 & 1.62 & 0.000 & 0.220 \\
\hline Up & Intracellular transport & 272 & 0.37 & 1.62 & 0.001 & 0.196 \\
\hline Up & Cellular localization & 360 & 0.36 & 1.62 & 0.000 & 0.194 \\
\hline Up & Sensory perception & 181 & 0.38 & 1.61 & 0.001 & 0.206 \\
\hline Up & Vesicle-mediated transport & 189 & 0.38 & 1.58 & 0.000 & 0.216 \\
\hline Up & Secretion & 175 & 0.38 & 1.57 & 0.000 & 0.213 \\
\hline Up & Organelle organization and biogenesis & 453 & 0.33 & 1.53 & 0.000 & 0.245 \\
\hline Down & Translation & 163 & -0.34 & -1.51 & 0.003 & 0.241 \\
\hline Down & Response to external stimulus & 297 & -0.33 & -1.58 & 0.000 & 0.216 \\
\hline Down & Immune system process & 304 & -0.34 & -1.61 & 0.000 & 0.194 \\
\hline Down & Regulation of protein modification process & 41 & -0.47 & -1.61 & 0.007 & 0.200 \\
\hline Down & Positive regulation of signal transduction & 123 & -0.38 & -1.63 & 0.000 & 0.181 \\
\hline Down & Regulation of immune system process & 64 & -0.43 & -1.68 & 0.003 & 0.158 \\
\hline Down & Response to wounding & 182 & -0.38 & -1.68 & 0.000 & 0.158 \\
\hline Down & Response to biotic stimulus & 107 & -0.41 & -1.69 & 0.000 & 0.155 \\
\hline Down & Regulation of protein amino acid phosphorylation & 28 & -0.54 & -1.70 & 0.005 & 0.159 \\
\hline Down & Cytokine- and chemokine-mediated signaling pathway & 22 & -0.58 & -1.73 & 0.009 & 0.127 \\
\hline Down & Developmental growth & 11 & -0.71 & -1.75 & 0.007 & 0.113 \\
\hline Down & Regulation of protein import into nucleus & 16 & -0.64 & -1.75 & 0.009 & 0.114 \\
\hline Down & Epithelial cell differentiation & 9 & -0.76 & -1.76 & 0.010 & 0.115 \\
\hline Down & Immune response & 214 & -0.38 & -1.76 & 0.000 & 0.120 \\
\hline Down & Inflammatory response & 123 & -0.42 & -1.76 & 0.000 & 0.128 \\
\hline Down & Multiorganism process & 134 & -0.41 & -1.76 & 0.000 & 0.134 \\
\hline Down & Regulation of immune response & 32 & -0.54 & -1.76 & 0.005 & 0.143 \\
\hline Down & Regulation of phosphorylation & 46 & -0.51 & -1.81 & 0.000 & 0.097 \\
\hline Down & Positive regulation of epithelial cell proliferation & 10 & -0.75 & -1.84 & 0.000 & 0.077 \\
\hline Down & Morphogenesis of an epithelium & 15 & -0.71 & -1.88 & 0.005 & 0.050 \\
\hline
\end{tabular}


TABLe 2: Continued.

\begin{tabular}{lcccccc}
\hline Direction in C3-def & Gene set & Size & ES & NES & NOM $p$ value & FDR $q$ value \\
\hline Down & Response to other organisms & 72 & -0.50 & -1.94 & 0.000 & 0.023 \\
Down & Tissue development & 133 & -0.47 & -1.97 & 0.000 & 0.016 \\
Down & Defense response & 239 & -0.43 & -1.98 & 0.000 \\
Down & Detection of biotic stimulus & 10 & -0.83 & -2.04 & 0.000 & 0.015 \\
Down & Acute inflammatory response & 11 & -0.85 & -2.05 & 0.000 & 0.010 \\
Down & Keratinocyte differentiation & 14 & -0.77 & -2.08 & 0.000 & 0.009 \\
Down & Response to bacterium & 28 & -0.67 & -2.12 & 0.000 & 0.001 \\
Down & Ectoderm development & 76 & -0.58 & -2.23 & 0.000 & 0.001 \\
Down & Epidermis development & 67 & -0.62 & -2.37 & 0.000 & 0.000 \\
\hline
\end{tabular}

susceptible to microbial infections [48]. According to the GO classification, biological processes that were mostly inhibited in aged C3-/- mice included the humoral immune response, immune response, defense response, humoral immune response mediated by circulating immunoglobulin, and immune system process. Simultaneously, GSEA allowed us to identify 29 gene sets that were negatively correlated to aged C3-deficient mice. Among them, the largest two groups are represented by gene sets associated with inflammation: acute inflammatory response, inflammatory response, cytokineand chemokine-mediated signaling pathway, and the immune system: response to bacterium, defense response, regulation of immune response, regulation of immune system process, and immune system process. Importantly, annotations involved in epithelial development and epithelial cell differentiation were significantly upregulated in WT mice, probably due to active mechanisms for countering the negative effects of oxidative stress on the RPE cells.

\section{Conclusions}

In summary, our study was designed to investigate the influence of complement component C3 global depletion on the biological structure and function of the aged retina. Aging is a significant risk factor for retinal degenerative diseases, and our collective data implicate $\mathrm{C} 3$ as potentially playing a role in the process of retinal degeneration. Importantly, the absence of active C3 resulted in decreased proapoptotic caspase-3 activity; elevated levels of antioxidant enzymes (catalase and glutathione reductase), autophagy-related ubiquitin, and cytosolic fraction of LC3B-II; and increased expression of several gene sets associated with maintaining physiological functions of the neural retina. All these changes potentially suggest that $\mathrm{C} 3$ inhibition may be protective against retinal degeneration. Our findings shed light on mechanisms of age-related retinal alterations identifying C3 as a potential therapeutic target for retinal aging. In particular, targeting the complement system may be promising for future therapeutic strategies in AMD disorders. Nevertheless, our observations were obtained in a model of systemic genetically depleted C3 in the whole organism and not selectively in ocular tissues. Therefore, further investigations should focus on targeted inhibition of $\mathrm{C} 3$ in the retina in various disease models to better understand the biological effects of C3 deficiency.

\section{Conflicts of Interest}

The authors declare that there is no conflict of interest regarding the publication of this article.

\section{Acknowledgments}

The authors are grateful to Mrs. Anne Seppänen for her expert technical assistance. This work was supported by the National Science Center Grant no. 2012/06/M/NZ4/00133 (to Bogusław Machaliński).

\section{References}

[1] M. Chen and H.Xu, "Parainflammation, chronic inflammation, and age-related macular degeneration," Journal of Leukocyte Biology, vol. 98, no. 5, pp. 713-725, 2015.

[2] I. Bhutto and G. Lutty, "Understanding age-related macular degeneration (AMD): relationships between the photoreceptor/retinal pigment epithelium/Bruch's membrane/choriocapillaris complex," Molecular Aspects of Medicine, vol. 33, no. 4, pp. 295-317, 2012.

[3] K. Kaarniranta, P. Tokarz, A. Koskela, J. Paterno, and J. Blasiak, "Autophagy regulates death of retinal pigment epithelium cells in age-related macular degeneration," Cell Biology and Toxicology, vol. 33, no. 2, pp. 113-128, 2017.

[4] K. Kaarniranta, D. Sinha, J. Blasiak et al., "Autophagy and heterophagy dysregulation leads to retinal pigment epithelium dysfunction and development of age-related macular degeneration," Autophagy, vol. 9, no. 7, pp. 973-984, 2013.

[5] N. Golestaneh, Y. Chu, Y. Y. Xiao, G. L. Stoleru, and A. C. Theos, "Dysfunctional autophagy in RPE, a contributing factor in age-related macular degeneration," Cell Death \& Disease, vol. 8, no. 1, article e2537, 2017.

[6] H. Y. Chung, M. Cesari, S. Anton et al., "Molecular inflammation: underpinnings of aging and age-related diseases," Ageing Research Reviews, vol. 8, no. 1, pp. 18-30, 2009.

[7] D. H. Cribbs, N. C. Berchtold, V. Perreau et al., "Extensive innate immune gene activation accompanies brain aging, increasing vulnerability to cognitive decline and neurodegeneration: a microarray study," Journal of Neuroinflammation, vol. 9, p. 179, 2012. 
[8] P. Herrmann, J. A. Cowing, E. Cristante et al., "Cd59a deficiency in mice leads to preferential innate immune activation in the retinal pigment epithelium-choroid with age," Neurobiology of Aging, vol. 36, no. 9, pp. 2637-2648, 2015.

[9] A. Machalińska, M. Kawa, E. Pius-Sadowska et al., "Long-term neuroprotective effects of NT-4-engineered mesenchymal stem cells injected intravitreally in a mouse model of acute retinal injury," Investigative Ophthalmology \& Visual Science, vol. 54, no. 13, pp. 8292-8305, 2013.

[10] W. Huang da, B. T. Sherman, and R. A. Lempicki, "Systematic and integrative analysis of large gene lists using DAVID bioinformatics resources," Nature Protocols, vol. 4, no. 1, pp. 44-57, 2009.

[11] A. Liberzon, C. Birger, H. Thorvaldsdottir, M. Ghandi, J. P. Mesirov, and P. Tamayo, "The molecular signatures database (MSigDB) hallmark gene set collection," Cell Systems, vol. 1, no. 6, pp. 417-425, 2015.

[12] A. Subramanian, P. Tamayo, V. K. Mootha et al., "Gene set enrichment analysis: a knowledge-based approach for interpreting genome-wide expression profiles," Proceedings of the National Academy of Sciences, vol. 102, no. 43, pp. 1554515550, 2005.

[13] M. Trejter, A. Hochol, M. Tyczewska et al., "Sex-related gene expression profiles in the adrenal cortex in the mature rat: microarray analysis with emphasis on genes involved in steroidogenesis," International Journal of Molecular Medicine, vol. 35, no. 3, pp. 702-714, 2015.

[14] S. Beatty, H. H. Koh, D. Henson, and M. Boulton, "The role of oxidative stress in the pathogenesis of age-related macular degeneration," Survey of Ophthalmology, vol. 45, no. 2, pp. 115-134, 2000.

[15] P. Tokarz, A. Kauppinen, K. Kaarniranta, and J. Blasiak, "Oxidative DNA damage and proteostasis in age-related macular degeneration," Journal of Biochemical and Pharmalogical Research, vol. 1, no. 2, pp. 106-113, 2013.

[16] J. Blasiak, G. Petrovski, Z. Vereb, A. Facsko, and K. Kaarniranta, "Oxidative stress, hypoxia, and autophagy in the neovascular processes of age-related macular degeneration," BioMed Research International, vol. 2014, Article ID 768026, 7 pages, 2014.

[17] S. M. Cohen, K. L. Olin, W. J. Feuer, L. Hjelmeland, C. L. Keen, and L. S. Morse, "Low glutathione reductase and peroxidase activity in age-related macular degeneration," The British Journal of Ophthalmology, vol. 78, no. 10, pp. 791-794, 1994.

[18] J. Cejkova, M. Vejrazka, J. Platenik, and S. Stipek, "Age-related changes in superoxide dismutase, glutathione peroxidase, catalase and xanthine oxidoreductase/xanthine oxidase activities in the rabbit cornea," Experimental Gerontology, vol. 39, no. 10, pp. 1537-1543, 2004.

[19] W. G. Robison Jr., T. Kuwabara, and J. G. Bieri, "Deficiencies of vitamins $\mathrm{E}$ and $\mathrm{A}$ in the rat. Retinal damage and lipofuscin accumulation," Investigative Ophthalmology \& Visual Science, vol. 19, no. 9, pp. 1030-1037, 1980.

[20] P. Zafrilla, M. Losada, A. Perez, G. Caravaca, and J. Mulero, "Biomarkers of oxidative stress in patients with wet age related macular degeneration," The Journal of Nutrition, Health \& Aging, vol. 17, no. 3, pp. 219-222, 2013.

[21] L. V. Johnson, S. Ozaki, M. K. Staples, P. A. Erickson, and D. H. Anderson, "A potential role for immune complex pathogenesis in drusen formation," Experimental Eye Research, vol. 70, no. 4, pp. 441-449, 2000.
[22] J. M. Thurman, B. Renner, K. Kunchithapautham et al., "Oxidative stress renders retinal pigment epithelial cells susceptible to complement-mediated injury," Journal of Biological Chemistry, vol. 284, no. 25, pp. 16939-16947, 2009.

[23] M. C. Marazita, A. Dugour, M. D. Marquioni-Ramella, J. M. Figueroa, and A. M. Suburo, "Oxidative stress-induced premature senescence dysregulates VEGF and CFH expression in retinal pigment epithelial cells: implications for age-related macular degeneration," Redox Biology, vol. 7, pp. 78-87, 2016.

[24] M. Chen, J. V. Forrester, and H. Xu, "Synthesis of complement factor $\mathrm{H}$ by retinal pigment epithelial cells is down-regulated by oxidized photoreceptor outer segments," Experimental Eye Research, vol. 84, no. 4, pp. 635-645, 2007.

[25] M. Tanito, M. H. Elliott, Y. Kotake, and R. E. Anderson, "Protein modifications by 4-hydroxynonenal and 4-hydroxyhexenal in light-exposed rat retina," Investigative Ophthalmology \& Visual Science, vol. 46, no. 10, pp. 3859-3868, 2005.

[26] S. K. Mitter, C. Song, X. Qi et al., "Dysregulated autophagy in the RPE is associated with increased susceptibility to oxidative stress and AMD," Autophagy, vol. 10, no. 11, pp. 1989-2005, 2014.

[27] A. L. Wang, T. J. Lukas, M. Yuan, N. Du, M. O. Tso, and A. H. Neufeld, "Autophagy, exosomes and drusen formation in agerelated macular degeneration," Autophagy, vol. 5, no. 4, pp. 563-564, 2009.

[28] A. Varshavsky, "The ubiquitin system," Trends in Biochemical Sciences, vol. 22, no. 10, pp. 383-387, 1997.

[29] T. Jung and T. Grune, "The proteasome and its role in the degradation of oxidized proteins," IUBMB Life, vol. 60, no. 11, pp. 743-752, 2008.

[30] N. Nedelsky, P. K. Todd, and J. P. Taylor, “Autophagy and the ubiquitin-proteasome system: collaborators in neuroprotection," Biochimica et Biophysica Acta, vol. 1782, no. 12, pp. 691-699, 2008.

[31] V. I. Korolchuk, A. Mansilla, F. M. Menzies, and D. C. Rubinsztein, "Autophagy inhibition compromises degradation of ubiquitin-proteasome pathway substrates," Molecular Cell, vol. 33, no. 4, pp. 517-527, 2009.

[32] Z. Zhao, Y. Chen, J. Wang et al., "Age-related retinopathy in NRF2-deficient mice," PLoS One, vol. 6, no. 4, article e19456, 2011.

[33] I. Johansson, V. T. Monsen, K. Pettersen et al., “The marine n3 PUFA DHA evokes cytoprotection against oxidative stress and protein misfolding by inducing autophagy and NFE2L2 in human retinal pigment epithelial cells," Autophagy, vol. 11, no. 9, pp. 1636-1651, 2015.

[34] L. Wang, K. B. Ebrahimi, M. Chyn, M. Cano, and J. T. Handa, "Biology of p62/sequestosome-1 in age-related macular degeneration (AMD)," Advances in Experimental Medicine and Biology, vol. 854, pp. 17-22, 2016.

[35] S. Pankiv, T. H. Clausen, T. Lamark et al., "p62/SQSTM1 binds directly to Atg8/LC3 to facilitate degradation of ubiquitinated protein aggregates by autophagy," The Journal of Biological Chemistry, vol. 282, no. 33, pp. 24131-24145, 2007.

[36] D. J. Klionsky, K. Abdelmohsen, A. Abe et al., "Guidelines for the use and interpretation of assays for monitoring autophagy (3rd edition)," Autophagy, vol. 12, no. 1, pp. 1-222, 2016.

[37] J. Viiri, M. Amadio, N. Marchesi et al., "Autophagy activation clears ELAVL1/HuR-mediated accumulation of SQSTM1/p62 during proteasomal inhibition in human retinal pigment epithelial cells," PLoS One, vol. 8, no. 7, article e69563, 2013. 
[38] N. Mizushima, “Autophagy: process and function,” Genes \& Development, vol. 21, no. 22, pp. 2861-2873, 2007.

[39] S. Selbonne, D. Francois, W. Raoul et al., "Protease nexin-1 regulates retinal vascular development," Cellular and Molecular Life Sciences, vol. 72, no. 20, pp. 3999-4011, 2015.

[40] D. Li and E. M. Ellis, "Aldo-keto reductase 7A5 (AKR7A5) attenuates oxidative stress and reactive aldehyde toxicity in V79-4 cells," Toxicology in Vitro, vol. 28, no. 4, pp. 707714, 2014

[41] G. Jia, R. Takahashi, Z. Zhang, Y. Tsuji, and H. Sone, “Aldo-keto reductase 1 family $B 7$ is the gene induced in response to oxidative stress in the livers of Long-Evans Cinnamon rats," International Journal of Oncology, vol. 29, no. 4, pp. 829-838, 2006.

[42] K. Reim, H. Regus-Leidig, J. Ammermuller et al., "Aberrant function and structure of retinal ribbon synapses in the absence of complexin 3 and complexin 4," Journal of Cell Science, vol. 122, Part 9, pp. 1352-1361, 2009.

[43] N. Brose, "For better or for worse: complexins regulate SNARE function and vesicle fusion," Traffic, vol. 9, no. 9, pp. 1403$1413,2008$.

[44] A. Stockman, H. E. Smithson, A. R. Webster et al., "The loss of the PDE6 deactivating enzyme, RGS9, results in precocious light adaptation at low light levels," Journal of Vision, vol. 8, no. 1, pp. 1-10, 2008.

[45] H. Patil, M. R. Guruju, K. I. Cho et al., "Structural and functional plasticity of subcellular tethering, targeting and processing of RPGRIP1 by RPGR isoforms," Biology Open, vol. 1, no. 2, pp. 140-160, 2012.

[46] B. Stevens, N. J. Allen, L. E. Vazquez et al., "The classical complement cascade mediates CNS synapse elimination," Cell, vol. 131, no. 6, pp. 1164-1178, 2007.

[47] Q. Shi, K. J. Colodner, S. B. Matousek et al., "Complement C3-deficient mice fail to display age-related hippocampal decline," Journal of Neuroscience, vol. 35, no. 38, pp. 1302913042, 2015.

[48] J. Bode, P. Dutow, K. Sommer et al., "A new role of the complement system: $\mathrm{C} 3$ provides protection in a mouse model of lung infection with intracellular Chlamydia psittaci," PLoS One, vol. 7, no. 11, article e50327, 2012. 


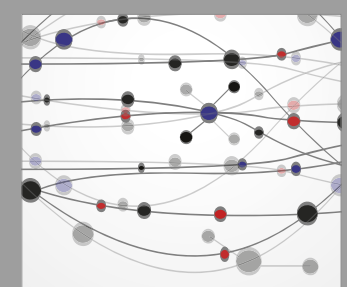

The Scientific World Journal
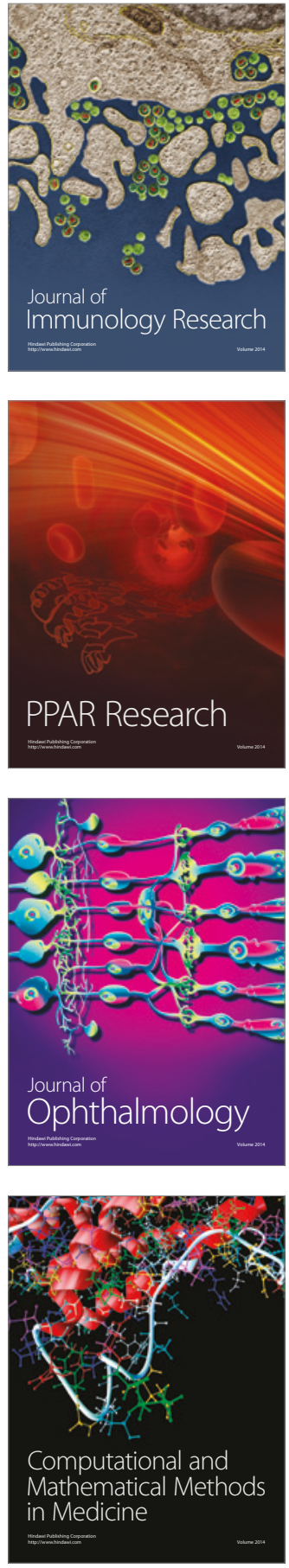

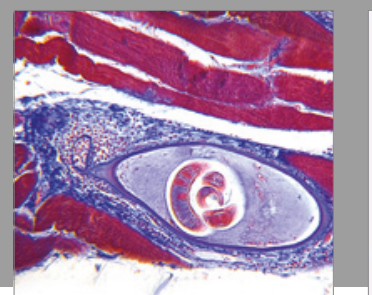

Gastroenterology Research and Practice
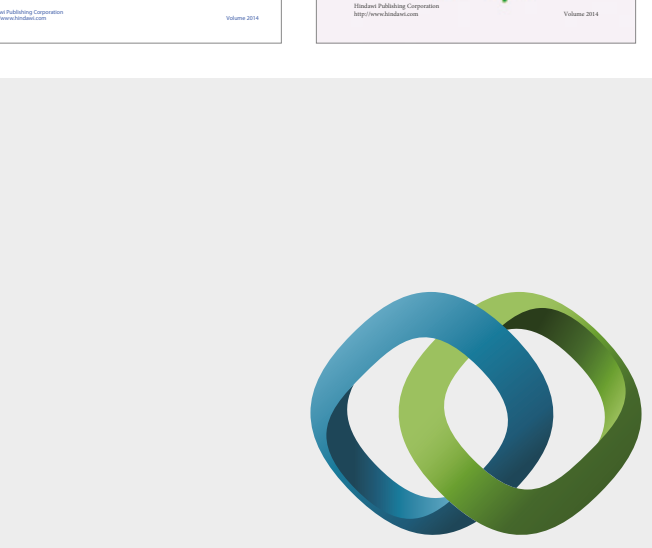

\section{Hindawi}

Submit your manuscripts at

https://www.hindawi.com
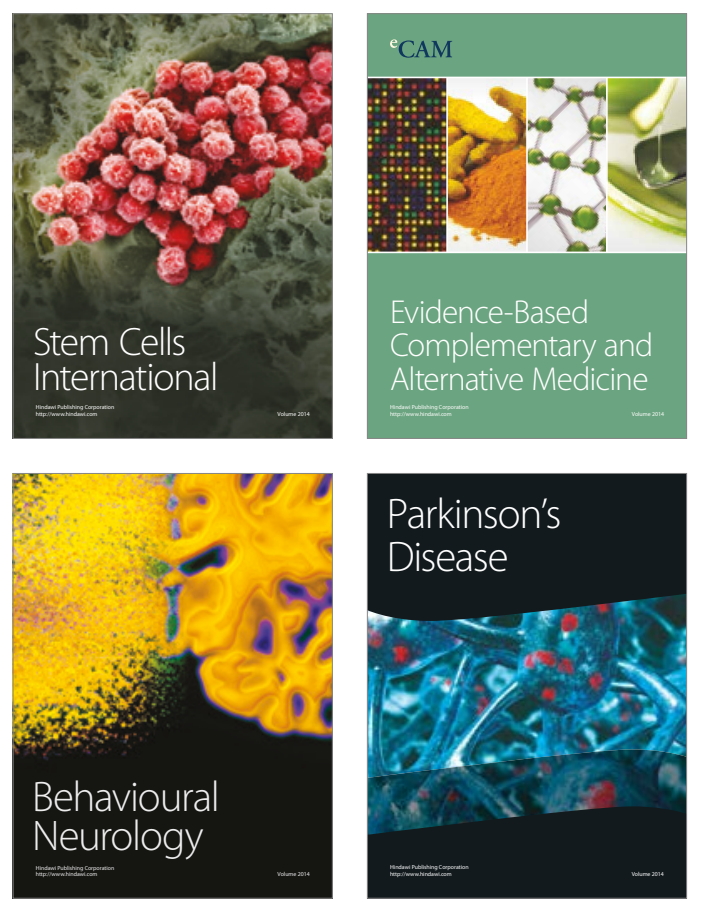
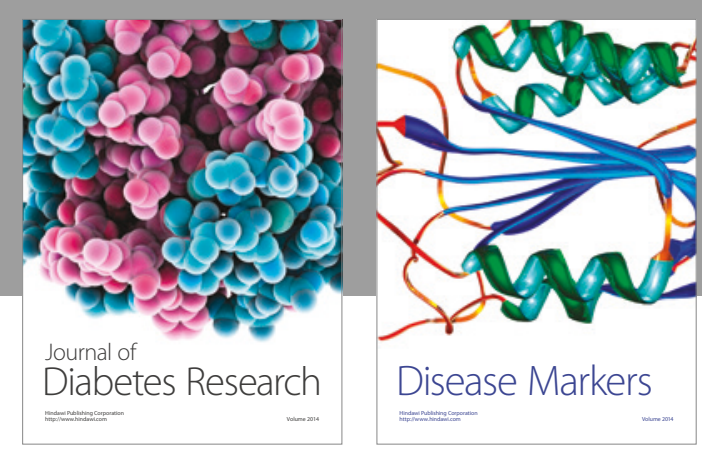

Disease Markers
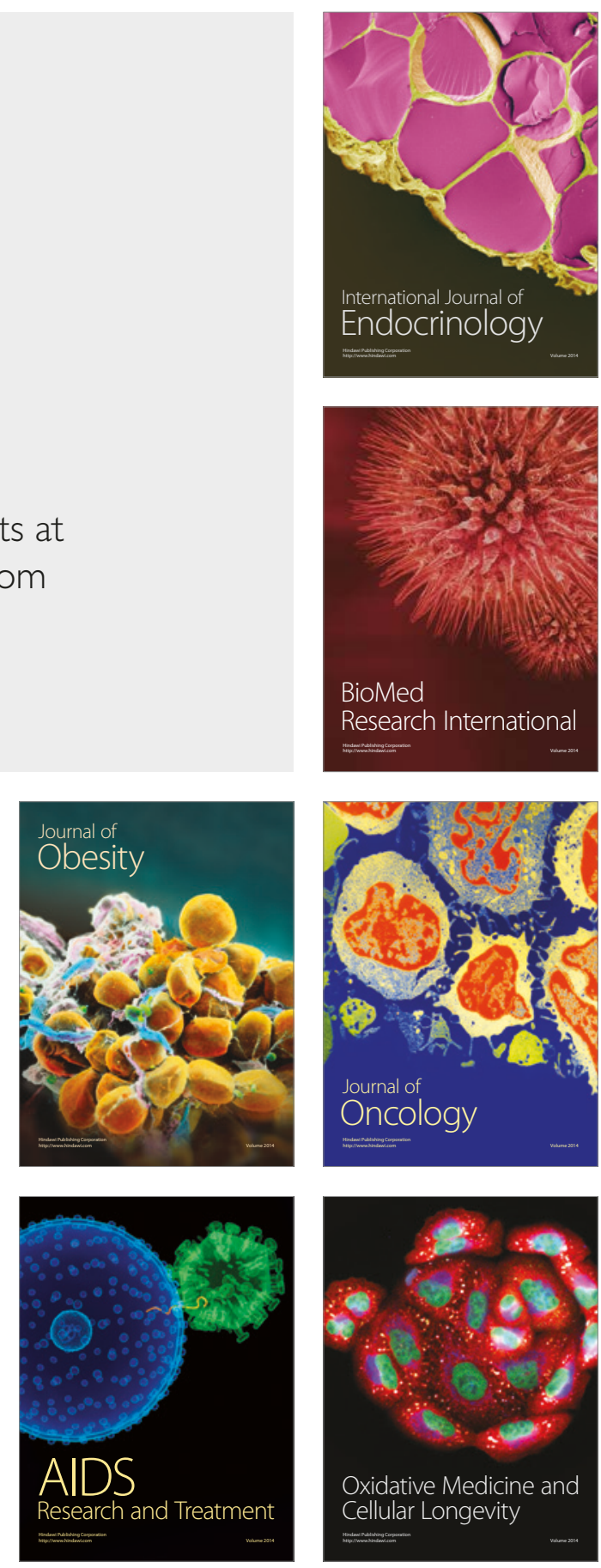